\title{
Woodward's Ambiguity Function: From Foundations to Applications
}

\author{
Dylan Eustice, Charles Baylis and Robert J. Marks II \\ Department of Electrical \& Computer Engineering \\ Baylor University, Waco, Texas \\ United States of America
}

\begin{abstract}
Woodward's ambiguity function, introduced in the literature in the mid-20th century, has been a staple topic in the study of radar performance. There exists an inherent trade-off in the ability of a signal to accurately measure both the range and velocity of a target. Woodward's ambiguity function measures this uncertainty for narrowband RF signals for monostatic radar. Despite its popularity and widespread description in both radar texts and the literature, there has been a wide variation in description of the ambiguity function. There are, for example, numerous similar albeit different definitions of the ambiguity function. There are also false statements made including claims that an ambiguity function is not invertable to its underlying signal and that best estimates of the range and Doppler of an object is found by analyzing the maximum of the magnitude of a correlation. In this paper, these and other questions are answered through derivation of the ambiguity function from first principles. Tables of ambiguity functions and their properties are presented, and 2-D Fourier analysis is shown to provide deeper insight into the ambiguity function structure.
\end{abstract}

\section{INTRODUCTION}

Woodward's ambiguity function [49], [50] describes the ability of a radar waveform $x(t)$ to uniquely specify the range and Doppler of a target. The literature is rich with application [2], [38], synthesis [17], [25], [27], [31], [45] and analysis [1], [7], [13]. The topic is a staple in most radar texts [11], [20], [21], [23], [39], [40], [43], [44].

Although there have been a number of tutorials on the topic [8], [32], [18], there remains significant deviation in the manner the ambiguity function is presented in the literature. There are, for example, a number of different definitions of the ambiguity function [14]. There are also unfortunate erroneous statements in the literature, including that the ambiguity function is not invertable (it is), the magnitude of the ambiguity function (rather than its real part) determines Doppler and range, and that the ambiguity function of the baseband signal is identical to that of the corresponding RF signal. (It's not.) [14].

Our goal is to develop in detail the ambiguity function from fundamentals and illustrate its proper interpretation. We derive the ambiguity function from basic principles. The function is shown to stem from intuitive minimum mean square error nearest neighbor principles. Tables of ambiguity function examples and properties are given. Finally, two dimensional Fourier analysis reveals interesting and useful properties of the ambiguity function in different domains.

\section{The Ambiguity Function}

The ambiguity of a possibly complex signal $x(t)$ can be defined as as [16], [21], [25], [27], [48]:

$$
\chi(\tau, u)=\int_{-\infty}^{\infty} x(t) x^{*}(t-\tau) e^{-j 2 \pi u t} d t
$$

where $\tau$ and $u$ are the range and Doppler errors relative to the actual range and Doppler of a measured target.

\section{DERIVATION}

The following is a derivation of the ambiguity function from first principles.

\section{A. Nearest Neighbor and Matched Filters}

Assume we have a library of $N$ possibly complex signals

$$
\left\{g_{n}(t) \mid 1 \leq n \leq N\right\}
$$

all of whom have the same energy. That is, for all $1 \leq n \leq N$,

$$
\left\|g_{n}(t)\right\|^{2}=C
$$

where the signal's energy is defined as

$$
\|h(t)\|^{2}:=\int_{t}|h(t)|^{2} d t .
$$

For an arbitrary signal, $f(t)$, which of these $N$ signals is closest? If we define closest in the mean square sense, we want to find the $g_{n}$ that minimizes the distance $\left\|f(t)-g_{n}(t)\right\|^{2}$. The best match to $f(t)$ (we'll call it $g^{\dagger}(t)$ ) is then

$$
g^{\dagger}(t)=\arg \min _{n}\left\|f(t)-g_{n}(t)\right\|^{2} .
$$

Since, for complex numbers $w$ and $z$,

$$
|w-z|^{2}=|w|^{2}+|z|^{2}-2 \Re w z^{*},
$$

where $\Re$ denotes "the real part of," this is equivalent to

$$
g^{\dagger}(t)=\arg \min _{n}\left(\|f(t)\|^{2}+\left\|g_{n}(t)\right\|^{2}-2 \int_{t} \Re f(t) g_{n}^{*}(t) d t\right) \text {. }
$$

Using the identical energy property in (3), the $\left\|g_{n}(t)\right\|^{2}$ term is the same for all $n$ and, when finding extrema, can be ignored. Likewise, $\|f(t)\|^{2}$ is just a nonnegative constant. The minimization thus occurs when $\Re f(t) g_{n}^{*}(t)$, the real part of the inner product of $f$ with $g$, is maximum. This recasts (5) as a 
maximization.

$$
g^{\dagger}(t)=\arg \max _{n}\left(\Re \int_{t} f(t) g_{n}^{*}(t) d t\right) .
$$

This is how the matched filter works. Maximizing the real part of the inner product of two signals is the same as minimizing the mean square error of the signals. ${ }^{1}$

\section{B. The Correlation Integral}

The elements in the matched filter library need not be isolated but can be positioned sequentially on a timeline. The signal $f(t)$ can then be slid along the timeline so that real part of the inner product is computed at every point. Let $f$ be shifted $\tau$ seconds. The inner product of $g(t)$ with $f(t-\tau)$ is the correlation integral

$$
g(\tau) \star f(\tau)=\int_{-\infty}^{\infty} g(t) f^{*}(t-\tau) d t .
$$

Correlation is related to convolution by the relationship

$$
g(t) \star f(t)=g(t) * f^{*}(-t) .
$$

To detect the presence of $f(t)$ in the signal $g(t)$ we wish to find those points that maximize $\Re(g(t) \star f(t))$.

Matched filter correlation is used extensively to detect signals in noise [9], [11], [20], [21], [28], [29], [42].

An illustration is shown in Figure 1. A sequence of \pm 1 height rectangular pulses is added to white Gaussian noise with unit variance. The encoded binary sequence of length $L=20$ is

$$
S=[11000010110010010110] \text {. }
$$

A bipolar representation of a string of bits merely replaces all of the 0's with -1 's. ${ }^{2}$

Pulse locations are obscured by the noisy signal

$$
g(t)=x(t)+\xi(t) .
$$

where $\xi(t)$ is the noise. Correlating the signal with the rectangular pulse gives the plot shown at the bottom of Figure 1. Peak values in the correlation correspond to the pulse locations and polarity. This alignment is illustrated more clearly in Figure 2. The decoding is simple. If the correlation is positive at a sample location, we announce a one. If negative, a zero. As is illustrated in Figure 2, we have successfully extracted the signal $S$ in (8) using a matched filter.

\footnotetext{
${ }^{1}$ The matched filter famously maximizes detection signal to noise ratio in the presence of white Gaussian noise. Minimizing the SNR is the basis of a more common derivation of the matched filter [46], [35].

${ }^{2}$ The bipolar form of $S$ is thus $B=2 S-1$. Denote the rectangular pulse by

$$
f(t)=\Pi\left(\frac{t}{a}-\frac{1}{2}\right)
$$

where $a$ is the pulse duration and the rectangle function is $\Pi(x)=1$ for $|x| \leq \frac{1}{2}$ and is otherwise zero. The replication of rectangular pulses can be written as

$$
x(t)=\sum_{\ell=1}^{L} B[\ell] f(t-\ell T)
$$
}

where $T$ is the pulse separation.
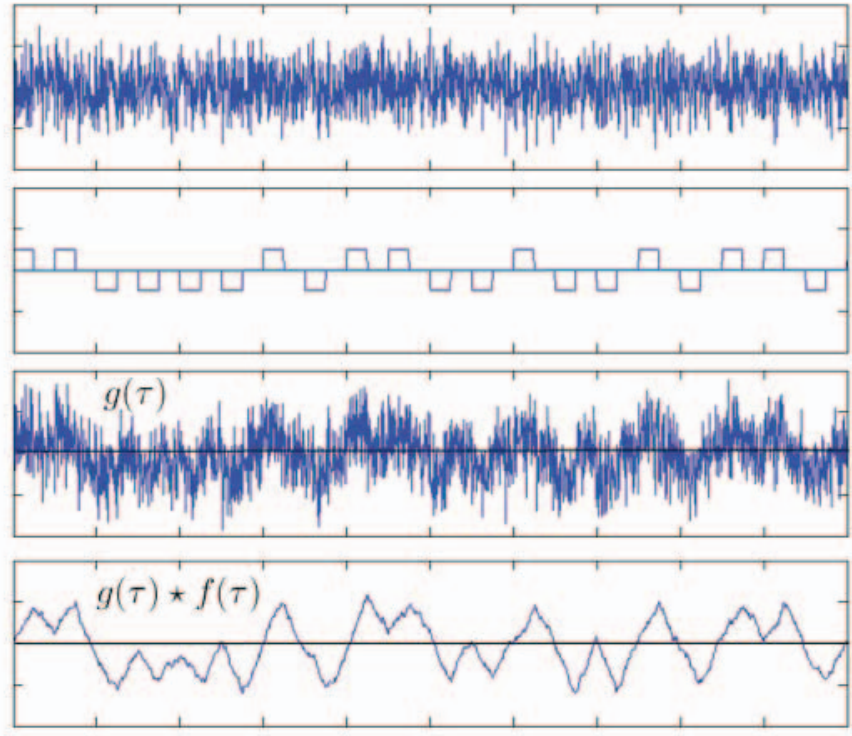

Fig. 1: White noise is added to a bipolar rectangular pulse train to give $g(t)$. Correlation of $g(t)$ with the pulse gives a correlation which peaks at positive pulse locations and dips negative for negative pulses. The triangular shapes indicating the pulse locations in the bottom plot are due to the choice of rectangular pulses to represent the signal. The autocorrelation of a rectangular pulse is a triangle function. (See (9)). The alignment of the autocorrelation with the pulses is illustrated in more detail in Figure 2.

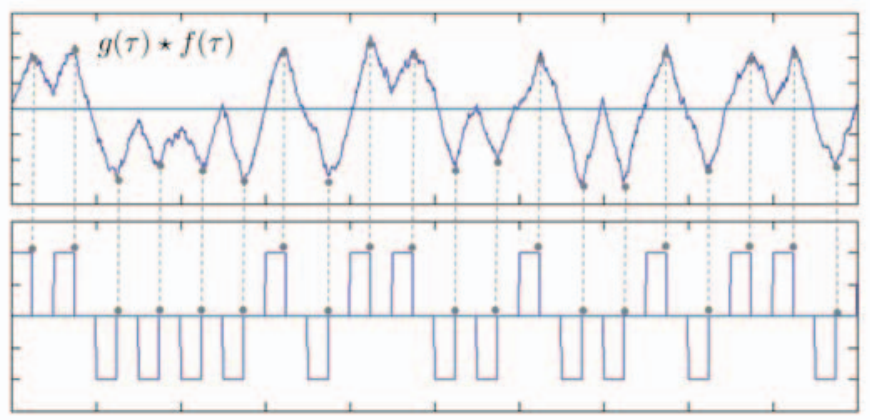

Fig. 2: The two plots here are identical to the bipolar rectangular pulse train and correlation plots in Figure 1. The peaks and valleys of the correlation are seen to align with the pulse train. Polarity of \pm 1 is also indicated. (The pulse $f(t)$ used in this example and Figure 1 has its right edge at $t=0$.) 

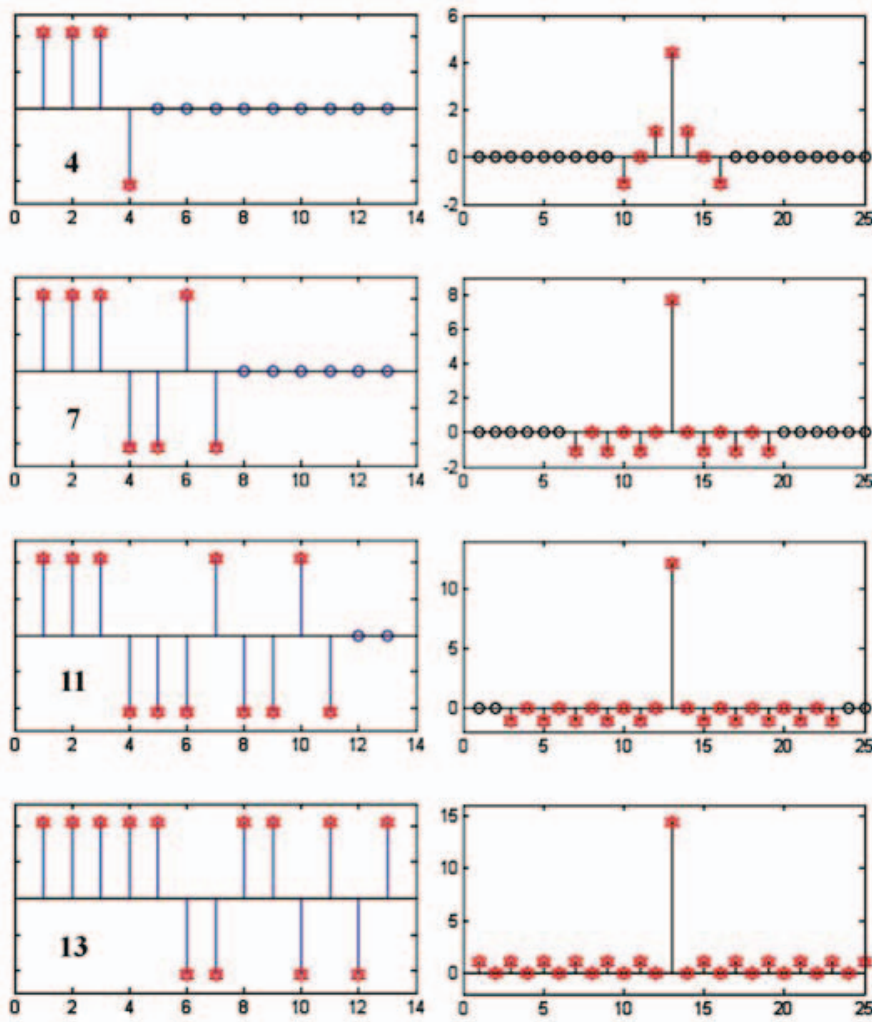

Fig. 3: Barker codes of length 4, 7, 11 and 13. The Barker code is shown on the left. The autocorrelation of the corresponding Barker code is shown on the right.

1) Choice of Signals: In Figures 1 and 2, a rectangular pulse is used for the encoding signal $f(t)$. If the pulse has amplitude $A$ and duration $a$, the rectangular pulse autocorrelation is the triangle function [30]

$$
A \Pi\left(\frac{t}{a}\right) \star A \Pi\left(\frac{t}{a}\right)=a A^{2} \Lambda\left(\frac{t}{a}\right)
$$

where $\Lambda(\xi)=1-|\xi|$ for $|\xi|<1$ and is otherwise zero. This is the reason that the pulse positions Figure 1 are indicated by triangular peaks and valleys. Ideally, we would like to have an encoding signal $f(t)$ with autocorrelation

$$
f(t) \star f(t)=\delta(t)
$$

where $\delta(\cdot)$ is the Dirac delta [31]. Choosing a signal with a good autocorrelation is directly analogous to choosing a signal with a good ambiguity function.

2) Barker Codes: There are a set of bit sequences called Barker codes [6] that are optimal in the sense that, besides the desired Dirac delta appearing peak at the origin, the remainder of the autocorrelation has a magnitude less than one. Ideally, to resemble the Dirac delta, the off peak values should be zero - but this is the best we can do with a finite duration encoding signal. A few Barker codes are shown in Figure 3. ${ }^{3}$ The longest known Barker code is of length $13 .^{4}$

If +1 is encoded as an 13 bit Barker code of pulses and -1 is encoded as its negative, the correlator will recover a bipolar signal bitstream better than when using only a single rectangular pulse as was done in Figures 1 and 2. Indeed, the correlation of the noisy signal in Figure 4(d) is so clean, that both the polarity and location of each pulse can be located by simple thresholding. In Figure 4(d), thresholds of \pm 0.9 are shown by horizontal gray lines.

The Barker code example in Figure 4 illustrates the performance of a good code. To illustrate ambiguity, let's examine a poorly performing 13 bit code that osicllates $1,-1,1,-1, \cdots$ for 13 places and ends with $a+1$. The coding of the sequence in (8) using this poorly performing code is shown on the left in Figure 5. When the coded sequence is correlated with the poorly performing code to recover the original signal, we obtain the correlation shown on the right in (8). Unlike the Barker code, decoding can not be done using a simple threshold operation. There are both positive and negative peaks as strong as those in the desired signal locations. These false peaks generate an ambiguity in the threshold decoding of the signal. This is not the ambiguity function, but illustrates that the choice of code impacts the ability to extract information from a noisy signal.

In temporal correlation, Barker codes are optimal. In simultaneous measurement of range and Doppler in radar signals, there is no single optimal signal to transmit. Rather, there is a tradeoff between range and Doppler resolution necessitating different choices of signals for varying performance requirements.

\section{The Ambiguity Function as a Matched Filter}

The Fourier transform a signal $g(t)$ is

$$
G(u)=\int_{-\infty}^{\infty} g(t) e^{-j 2 \pi u t} d t
$$

and will be denoted by a double arrow

$$
g(t) \longleftrightarrow G(u) .
$$

We are given a possibly complex baseband signal, $g(t)$, with bandwidth $B$. In other words,

$$
g(t) \longleftrightarrow G(u)=0 \text { for }|u|>B
$$

Define the narrowband (RF) signal

$$
\begin{aligned}
s_{W}(t) & =\Re g(t) e^{j 2 \pi W t} \\
& =|g(t)| \cos (2 \pi W t+\angle g(t))
\end{aligned}
$$

where $W$ is a carrier frequency. We will require in the specific sense

$$
W \gg B
$$

\footnotetext{
3 The Barker code of length 11 shown in Figure 3 is used in the IEEE 802.11b wireless local area network (WLAN) Standard [33], [19].

${ }^{4}$ It has been proven that there are no further odd length Barker codes,[47] nor even length codes less than 1022. [22]
} 

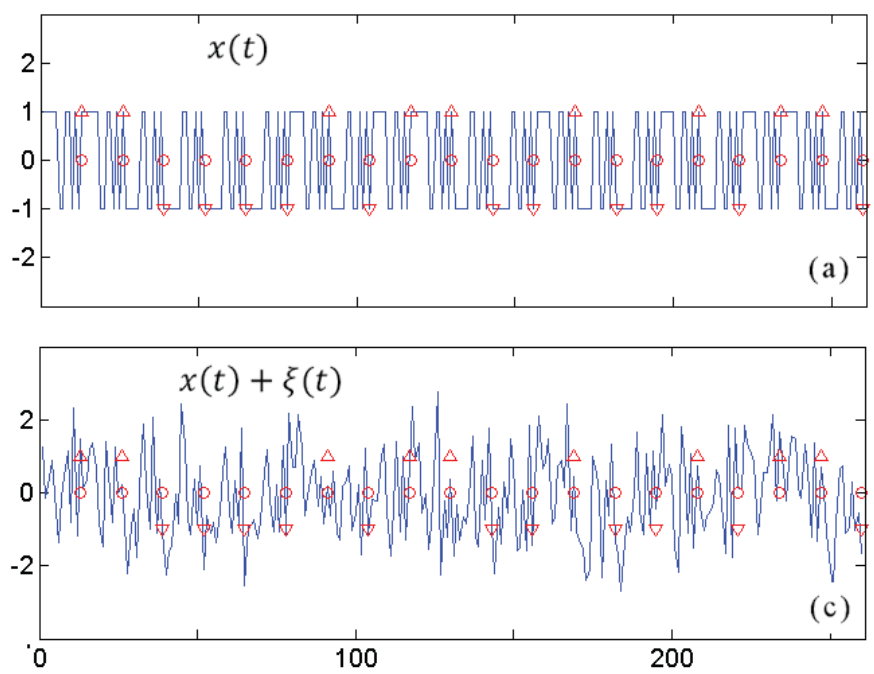
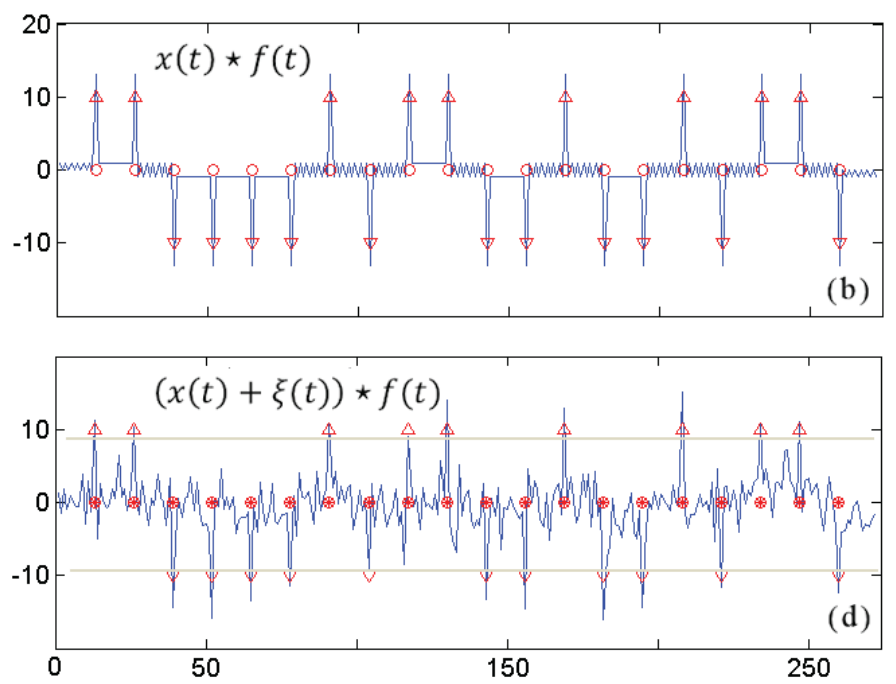

Fig. 4: In (a), the bipolar version of the sequence in (8) is shown encoded as $x(t)$ using a Barker code of length 13. This is the same binary sequence used in Figures 1 and 2. The Barker code of length 13 shown on the bottom left in Figure 3. For a one, the Barker code is used. For a zero, the negative of the Barker code is used. The axis markers in all four figures are located at the end of each pulse. Arrow heads indicate whether the code is \pm 1 . The correlation of $x(t)$ with the 13 length Barker code is shown in (b). The correlation is nicely peaked at the desired locations. The signal $x(t)$ is corrupted in (c) by additive zero mean white Gaussian noise, $\xi(t)$, with a variance of $\frac{1}{2}$. Since the Barker pulses are always \pm 1 , the signal level is one and the overall SNR is 2. As is shown in (d), the correlation of the noisy signal still peaks well at the desired locations allowing identification of the pulse as \pm 1 . )

Under this condition, we show in Appendix VII-A1 that

$$
\left\|s_{W}(t)\right\|^{2}=\frac{1}{2}\|g(t)\|^{2}
$$

The narrowband signals has twice the energy of the baseband signal.

1) Transmitted and Received: A transmitted signal $s_{W}(t)$ is reflected and received as

$$
\begin{gathered}
s_{W+\Delta W}(t-\Delta t)=\Re g(t-\Delta t) e^{j 2 \pi(W+\Delta W)(t-\Delta t)} \\
=|g(t-\Delta t)| \cos (2 \pi(W+\Delta W)(t-\Delta t)+\angle g(t-\Delta t))
\end{gathered}
$$

where the unknown Doppler shift is $\Delta W$ and the unknown time delay is $\Delta t$. To find $\Delta t$ and $\Delta W$, we compare this signal to

$s_{W-\nu}(t-\tau)=|g(t-\tau)| \cos (2 \pi(W-\nu)(t-\tau)+\angle g(t-\tau))$

for all possible $\tau$ and $\nu$. Our library of signals is no longer indexed by an integer $n$ that indicates a specific signal in a matched filter bank, but consists of a continuum of values. To identify both the unknown Doppler shift $\Delta W$ and time delay $\Delta t$, we calculate the mean square difference between the received signal in (14) and (15) for all $\tau$ and $\nu$ and see which gives the smallest value. Like the derivation of the matched filter, finding $\tau$ and $\nu$ to minimize the square error

$$
\min _{\tau, \nu}\left\|s_{W-\Delta W}(t-\Delta t)-s_{W-\nu}(t-\tau)\right\|^{2}
$$

is the same as finding $\tau$ and $\nu$ to maximize the real component of the inner product

$$
\Re e^{-j 2 \pi(W+\nu)(\Delta t-\tau)} \chi(\tau-\Delta t, \nu-\Delta W)
$$

In other words,

$$
\begin{aligned}
(\Delta t, \Delta W)= & \arg \min _{\tau, \nu}\left\|s_{W+\Delta W}(t-\Delta t)-s_{W+\nu}(t-\tau)\right\|^{2} \\
= & \arg \max _{\tau, \nu} \Re\left[e^{-j 2 \pi(W+\nu)(\Delta t-\tau)}\right. \\
& \left.\times \chi_{g}(\tau-\Delta t, \nu-\Delta W)\right]
\end{aligned}
$$

where $\chi_{g}$ is the ambiguity function of $g$ as defined in (1). This result, proven in Appendix VII-B2, shows that the ambiguity function, $\chi_{g}$, arises naturally in the matched filtering operation when identifying both range and Doppler.

2) Ambiguity Function and Envelope Maximization Are Almost the Same Thing.: The term to maximize in (16) is

$$
\Re\left[e^{-j 2 \pi(W+\nu)(\Delta t-\tau)} \chi(\tau-\Delta t, \nu-\Delta W)\right]
$$

For $W \gg B$, this is a high frequency sinusoid with an envelope of

$$
|\chi(\tau-\Delta t, \nu-\Delta W)| .
$$

Rather than maximize the oscillation details where local minima and maxima are closely spaced, we maximize the envelope, i.e. maximize

$$
|\chi(\tau-\Delta t, \nu-\Delta W)|
$$



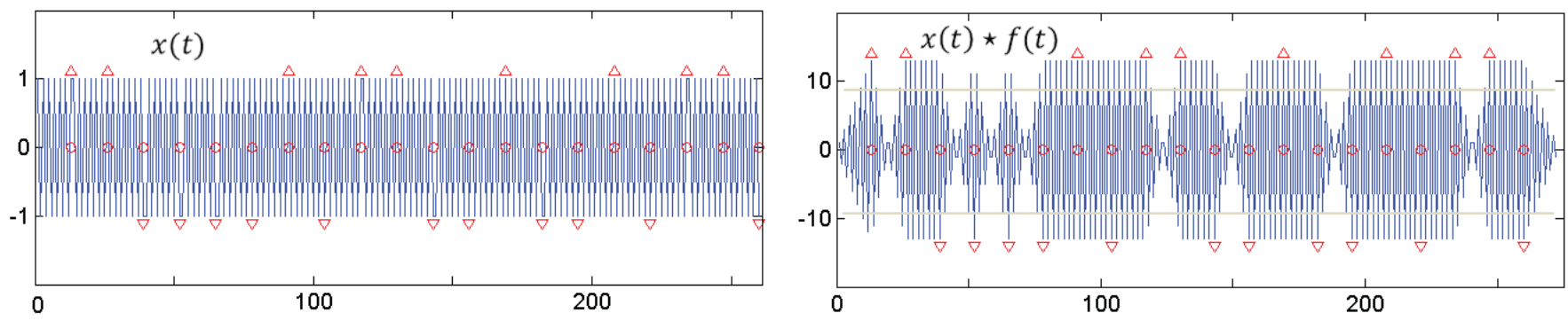

Fig. 5: The Barker code in Figure 4 is a good code choice. Illustrated here is a poorly performing code choice. The correlation on the right presents multiple positive and negative peaks thereby introducing ambiguity into the decoding process when using simple threshold decoding. The SNR here is the same as that used for the Barker code example in the bottom two plots in Figure 4.

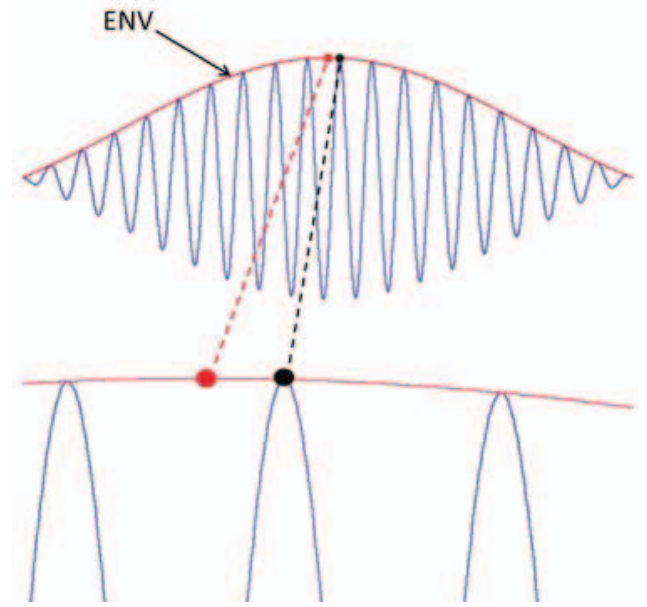

Fig. 6: Illustration that maximizing the highly oscillatory function in (17) is approximately the same as maximizing the envelope function in (18). In the top figure, the red curve marked ENV corresponds to $|\chi|$ in (18) whereas the oscillation corresponds to the $\Re\left(e^{j \text { shift }} e^{-j \text { frequency } t} \chi\right)$ term in (17). The maximum of the envelope, denoted by the red dot, is close to the maximum of the oscillatory signal. The bottom figure zooms in on these points. The maxima are separated by no more that a period of the oscillation. At radio frequencies, this period is very short and the two maxima are equivalent for all practical purposes.

As is illustrated in Figure 6, doing so results in almost the same result. Thus, in lieu of (16),

$$
(\Delta t, \Delta W) \approx \arg \max _{\tau, \nu}\left|\chi_{g}(\tau-\Delta t, \nu-\Delta W)\right|
$$

The maximization occurs when $\tau=\Delta t$ and $\nu=\Delta W$.

3) Ambiguity.: Consider again one dimensional correlation. If the rectangle is correlated with another rectangle located somewhere on the time axis, the result is a triangle function centered where the second rectangle is located. The triangle is a property of the function chosen for the correlation kernel, in this case a rectangle. If we had chosen another function instead of the rectangle as a kernel, we would not have a triangle function, e.g. optimal Barker codes or the poorly performing code illustrated in Figure 5.

The ambiguity function works the same way except the ambiguity function of $g(t)$ replaces the autocorrelation of the rectangular pulses or Barker code. Perhaps the most exciting property of the solution in (19) is not that the function peaks at the desired optimal solution $(\Delta t, \Delta W)$, but that, no matter where the optimal solution lies on the $(\tau, \nu)$ plane, the matched filter for the narrowband signal, $s_{W}$, specified uniquely by the baseband signal, $g$, and carrier frequency, $W$, is totally defined by the ambiguity function $\chi_{g}$. This is illustrated in Figure 7 . Note the following properties.

1) $\left|\chi_{g}\right|$ will be centered at the optimal solution in the $(\tau, \nu)$ plane but does not change shape with respect to the measured Doppler and range, i.e. with $\Delta W$ and $\Delta t$,

2) $\left|\chi_{g}\right|$ is not a function of the carrier frequency, $W$, and

3) $\left|\chi_{g}\right|$ is only a function of $g$.

Thus, the properties of the ambiguity function $\chi_{g}$ spawned from a $g$ are worthy of study. Of particular interest is the case where there are other peaks in $\left|\chi_{g}\right|$ that may have values close to ideal. Such false maxima can occur when the returned signal is accompanied by noise and clutter. When another peak has a value close to that which is optimal, there is ambiguity at which point the optimal occurs. For this reason, $\chi_{g}$ and sometimes $\left|\chi_{g}\right|$ are referred to as ambiguity functions.

\section{Ambiguity Function Properties}

The ambiguity function obeys many interesting and useful properties. To facititate discussion, we will adopt the notation of the mapping of a signal to an ambiguity function in (1) by

$$
x(t) \Rightarrow \chi(\tau, u) .
$$

In some instances, specific reference to the signal will be made using a subscript, i.e.

$$
x(t) \Rightarrow \chi_{x}(\tau, u) .
$$

or even more explicitly,

$$
x(t) \Rightarrow \chi_{x(t)}(\tau, u) .
$$



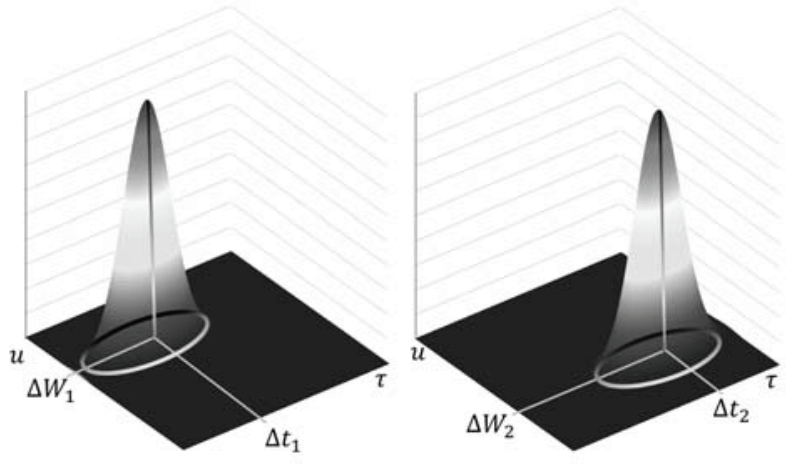

Fig. 7: The correlation for all range values, $\tau$ and all Doppler shifts, $u$, gives a matched filter output that peaks at the target's true range, $\Delta t$ and Doppler $\Delta W$. Shown here are the results of two cases. Independent of the measured range and Doppler, the function centered around the measured values always has the same shape as shown here. This invariant shape, when shifted to the origin, is the ambiguity function, $\chi(\tau, u)$. The shape of the ambiguity function is determined solely by the signal $g$.

a) The Origin of the Ambiguity Function is the Signal Energy: The energy of the signal $x(t)$ is equal to the origin of the ambiguity function.

$$
\chi(0,0)=\|x(t)\|^{2} .
$$

The proof follows immediately from the definition of the ambiguity function in (1).

b) Ambiguity Function in Terms of Fourier Transforms: The ambiguity function can be written in terms of a signal's Fourier transform

$$
\chi(\tau, u)=\int_{-\infty}^{\infty} X(f+u) X^{*}(f) e^{j 2 \pi f \tau} d f
$$

where $x(t) \longleftrightarrow X(u)$ or, equivalently by setting $\nu=f+u$,

$$
\chi(\tau, u)=e^{-j 2 \pi u \tau} \int_{-\infty}^{\infty} X(\nu) X^{*}(\nu-u) e^{j 2 \pi \nu \tau} d \nu
$$

Proof: The ambiguity function in (20) can be written as

$$
\chi(\tau, u)=\int_{-\infty}^{\infty}\left(x(t) e^{-j 2 \pi u t}\right)(x(t-\tau))^{*} d t
$$

Let $f$ denote the Fourier transform variable for $t$. From the modulation theorem of Fourier analysis [31],

$$
x(t) e^{-j 2 \pi u t} \longleftrightarrow X(f+u) .
$$

and from the shift theorem [31],

$$
x(t-\tau) \longleftrightarrow X(f) e^{-j 2 \pi f \tau} .
$$

Applying these relationships to (22) and applying the power theorem [31] gives the expression in (20).

\section{A. Ambiguity Functions of Some Signals}

A short table of ambiguity functions is in Table I. Most can be derived using the properties and theorems of the Fourier transform [31].

1) Dirac delta: When

$$
x(t)=\delta(t-\xi),
$$

application of (1) using the sifting property of the Dirac delta gives

$$
\begin{aligned}
\delta(t-\xi) \Rightarrow \chi(\tau, u) & =\int_{-\infty}^{\infty} \delta(t-\xi) \delta((t-\xi)-\tau) e^{j 2 \pi t u} d t \\
& =\delta(\tau) e^{j 2 \pi \xi u}
\end{aligned}
$$

which is a Dirac delta sheet along the $u$ axis.

2) Complex Sinusoid: Let

$$
x(t)=e^{j 2 \pi W t} .
$$

Then (1) gives

$$
\begin{aligned}
e^{j 2 \pi W t} \Rightarrow \chi(\tau, u) & =\int_{-\infty}^{\infty} e^{j 2 \pi W t} e^{-j 2 \pi W(t-\tau)} e^{-j 2 \pi u t} d t \\
& =\int_{-\infty}^{\infty} e^{-j 2 \pi(u-W) t} d t \\
& =\delta(u-W)
\end{aligned}
$$

which is a Dirac delta sheet for $u=W$ independent of $\tau$.

3) Real Sinusoid: To evaluate the ambiguity function for the real sinusoid we first take the Fourier transform

$x(t)=\cos (2 \pi W t) \longleftrightarrow X(f)=\frac{1}{2}[\delta(f-W)+\delta(f+W)]$.

Substituting into (20) gives

$$
\begin{aligned}
\chi(\tau, u)= & \frac{1}{4} \int_{-\infty}^{\infty}[\delta(f+u-W)+\delta(f+u+W)] \\
& {[\delta(f-W)+\delta(f+W)] e^{j 2 \pi f \tau} d f } \\
= & \frac{1}{4}\left[2 \cos (2 \pi W \tau) \delta(u)+e^{-j 2 \pi W \tau} \delta(u-2 W)\right. \\
& \left.+e^{j 2 \pi W \tau} \delta(u+2 W)\right]
\end{aligned}
$$

which is the result in Table I.

4) Gaussian.: To prove the entry for the Gaussian $x(t)=$ $e^{-\pi(\varphi t)^{2}}$ in Table I, we begin by substituting into the ambiguity function expression in (1).

$$
\begin{aligned}
\chi(\tau, u) & =\int_{-\infty}^{\infty} e^{-\pi(\varphi t)^{2}} e^{-\pi(\varphi(t-\tau))^{2}} e^{-j 2 \pi u t} d t \\
& =e^{-\pi(\varphi \tau)^{2}} \int_{-\infty}^{\infty} e^{-2 \pi \varphi^{2}\left(t^{2}-t \tau\right)} e^{-j 2 \pi u t} d t
\end{aligned}
$$

Completing the square

$$
t^{2}-t \tau+\left(\frac{\tau}{2}\right)^{2}=\left(t-\frac{\tau}{2}\right)^{2}
$$




\begin{tabular}{|l|rl|}
\hline Signal & $x(t)$ & $\Rightarrow \chi_{x}(\tau, u)$ \\
\hline \hline Dirac delta & $\delta(t-\lambda)$ & $\Rightarrow \delta(\tau) e^{j 2 \pi \lambda u}$ \\
complex sinusoid & $e^{j 2 \pi W t}$ & $\Rightarrow \delta(u-W)$ \\
rectangular pulse & $\Pi\left(\frac{t}{2 T}\right)$ & $\Rightarrow e^{-j \pi u \tau}|2 T-| \tau|| \operatorname{sinc}((2 T-|\tau|) u) \Pi\left(\frac{\tau}{4 T}\right)$ \\
sinc pulse & $\operatorname{sinc}(2 B t)$ & $\Rightarrow e^{-j \pi u \tau}\left(\frac{a}{2 B}\right)^{2}|2 B-| u|| \times \operatorname{sinc}((2 B-|u|) \tau) \Pi\left(\frac{u}{4 B}\right)$ \\
real sinusoid & $\cos (2 \pi W t)$ & $\Rightarrow \frac{1}{4}\left[2 \cos (2 \pi W \tau) \delta(u)+e^{-j 2 \pi W \tau} \delta(u-2 W)+e^{j 2 \pi W \tau} \delta(u+2 W)\right]$ \\
complex chirp & $e^{j \pi \alpha t^{2}}$ & $\Rightarrow e^{-j \pi \alpha \tau^{2}} \delta(u-\alpha \tau)$ \\
Gaussian & $e^{-\pi(\varphi t)^{2}}$ & $\Rightarrow \frac{1}{\sqrt{2}|\varphi|} e^{-\frac{\pi(\varphi \tau)^{2}}{2}} e^{-\frac{\pi}{2}\left(\frac{u}{\varphi}\right)^{2}} e^{-j \pi u \tau}$ \\
\hline
\end{tabular}

TABLE I: A table of some signals and their corresponding ambiguity functions derived in Section IV-A. $\lambda, \alpha$ and $\varphi$ are real (i.e. $\in \mathbb{R}$ ), whereas $T, W$ and $B$ are positive.

gives

$$
\begin{aligned}
\chi(\tau, u) & =e^{-\pi(\varphi \tau)^{2}} \int_{-\infty}^{\infty} e^{-2 \pi \varphi^{2}\left(\left(t-\frac{\tau}{2}\right)^{2}-\frac{\tau^{2}}{4}\right)} e^{-j 2 \pi u t} d t \\
& =e^{-\frac{\pi}{2}(\varphi \tau)^{2}} \int_{-\infty}^{\infty} e^{-2 \pi \varphi^{2}\left(t-\frac{\tau}{2}\right)^{2}} e^{-j 2 \pi u t} d t
\end{aligned}
$$

Recall that the Fourier transform of a Gaussian is a Gaussian [31]: $e^{-\pi t^{2}} \leftrightarrow e^{-\pi u^{2}}$. Thus, applying the scaling theorem [31]

$$
\begin{aligned}
e^{-2 \pi(\varphi t)^{2}} \longleftrightarrow & \int_{-\infty}^{\infty} e^{-2 \pi \varphi^{2} t^{2}} e^{-j 2 \pi u t} d t \\
& =\frac{1}{\sqrt{2} \varphi} e^{-\frac{\pi}{2}\left(\frac{u}{\varphi}\right)^{2}} .
\end{aligned}
$$

and then the shift theorem [31] gives

$$
e^{-2 \pi \varphi^{2}\left(t-\frac{\tau}{2}\right)^{2}} \longleftrightarrow \frac{1}{\sqrt{2} \varphi} e^{-\frac{\pi}{2}\left(\frac{u}{\varphi}\right)^{2}} e^{-j \pi \tau u}
$$

Applying to (24) gives the entry in Table I.

$$
\chi(\tau, u)=\frac{1}{\sqrt{2} \varphi} e^{-\frac{\pi(\varphi \tau)^{2}}{2}} e^{-\frac{\pi u^{2}}{2 \varphi}} e^{-j \pi u \tau}
$$

The contours for $|\chi(\tau, u)|$ are concentric ellipses corresponding to the family of curves

$$
(\varphi \tau)^{2}+\left(\frac{u}{\varphi}\right)^{2}=\text { constant. }
$$

5) Rectangular Pulse:: For the rectangular pulse, $x(t)=$ $\Pi\left(\frac{t}{2 T}\right)$, the ambiguity function is

$$
\begin{aligned}
\chi(\tau, u) & =\int_{-\infty}^{\infty} \Pi\left(\frac{t}{2 T}\right) \Pi\left(\frac{t-\tau}{2 T}\right) e^{-j 2 \pi u t} d t \\
& =\int_{-T}^{T} \Pi\left(\frac{t-\tau}{2 T}\right) e^{-j 2 \pi u t} d t
\end{aligned}
$$

For $0 \leq \tau \leq 2 T$,

$$
\begin{aligned}
\chi(\tau, u) & =\int_{-T+\tau}^{T} e^{-j 2 \pi u t} d t \\
& =\int_{-\infty}^{\infty} \Pi\left(\frac{t-\frac{\tau}{2}}{2 T-\tau}\right) e^{-j 2 \pi u t} d t \\
& =|2 T-\tau| \operatorname{sinc}((2 T-\tau) u) e^{-j \pi u \tau} ; 0 \leq \tau \leq 2 T
\end{aligned}
$$

Similarly, for $-2 T \leq \tau \leq 0$,

$$
\begin{aligned}
\chi(\tau, u) & =\int_{-T}^{\tau+T} e^{-j 2 \pi u t} d t \\
& =|2 T+\tau| \operatorname{sinc}((2 T+\tau) u) e^{-j \pi u \tau} ;-2 T \leq \tau \leq 0
\end{aligned}
$$

and we get the corresponding entry in the result in Table I.

\section{B. Ambiguity Function Properties}

Some properties of the ambiguity function are summarized in Table II. Here are derivations of some of the less obvious entries.

1) Temporal Correlation: Along the $\tau$ axis, the ambiguity function is the autocorrelation of $x(t)$.

$$
\begin{aligned}
\chi(\tau, 0) & =x(\tau) \star x(\tau) \\
& =\int_{-\infty}^{\infty} x(t) x^{*}(t-\tau) d t
\end{aligned}
$$

This follows from setting $u=0$ in the ambiguity function in (1).

2) Spectral Correlation.: Likewise, along the $u$ axis, the ambiguity function is the autocorrelation of the spectrum of $x(t)$.

$$
\begin{aligned}
\chi(0, u) & =X(u) \star X(u) \\
& =\int_{-\infty}^{\infty} X(\nu) X^{*}(\nu-u) d \nu
\end{aligned}
$$

This follows from setting $\tau=0$ in the ambiguity function expression in (21).

3) Ambiguity Function Origin: The origin of the ambiguity function is the signal energy.

$$
\begin{aligned}
\chi(0,0) & =\|x(t)\|^{2}=\int_{-\infty}^{\infty}|x(t)|^{2} d t \\
& =\|X(u)\|^{2}=\int_{-\infty}^{\infty}|X(u)|^{2} d u .
\end{aligned}
$$

Equation (28) follows from (26) and (29) from (27) for $u=0 .{ }^{5}$

4) Ambiguity Function Area:

$$
\int_{\tau} \int_{u} \chi(\tau, u) d \tau d u=x(0) X^{*}(0)
$$

\footnotetext{
${ }^{5}$ Equivalently, (29) follows from (28) and Parseval's theorem.
} 


\begin{tabular}{|c|c|}
\hline Property & Ambiguity Function Properties \\
\hline Definition & $x(t) \Rightarrow \chi_{x}(\tau, u)$ \\
\hline $\begin{array}{l}\text { Quadratic } \\
\text { Homog. }\end{array}$ & $a x(t) \Rightarrow|a|^{2} \chi_{x}(\tau, u)$ \\
\hline Conjugate & $x^{*}(t) \Rightarrow \chi_{x}^{*}(\tau,-u)$ \\
\hline Transpose & $x(-t) \Rightarrow \chi_{x}(\tau,-u)$ \\
\hline $\begin{array}{l}\text { Conjugate } \\
\text { Transpose }\end{array}$ & $x^{*}(-t) \Rightarrow \chi_{x}^{*}(\tau, u)$ \\
\hline Shift & $x(t-T) \Rightarrow e^{-j 2 \pi u T} \chi_{x}(\tau, u)$ \\
\hline Scale & $x\left(\frac{t}{S}\right) \Rightarrow|S| \chi_{x}\left(\frac{\tau}{S}, S u\right)$ \\
\hline $\begin{array}{l}\text { Temporal } \\
\text { Corr. }\end{array}$ & $\chi(\tau, 0)=x(\tau) \star x(\tau)$ \\
\hline $\begin{array}{l}\text { Spectral } \\
\text { Corr. }\end{array}$ & $\chi(0, u)=X(u) \star X(u)$ \\
\hline AF Area & $\int_{\tau} \int_{u} \chi_{x}(\tau, u) d \tau d u=x(0) X^{*}(0)$ \\
\hline AF Energy & $\int_{\tau} \int_{u}\left|\chi_{x}(\tau, u)\right|^{2} d \tau d u=\left|\chi_{x}(0,0)\right|^{2}$ \\
\hline $\begin{array}{l}\text { Symmetry } \\
\text { (General) }\end{array}$ & $\chi^{*}(-\tau, u)=\chi(\tau,-u) e^{-j 2 \pi u \tau}$ \\
\hline $\begin{array}{l}\text { Symmetry } \\
\quad(\text { Real } x(t))\end{array}$ & $\chi(\tau, u)=\chi^{*}(\tau,-u)$ \\
\hline $\begin{array}{l}\text { Preservation } \\
\text { Frequency }\end{array}$ & $x(t) e^{j 2 \pi W t} \Rightarrow \chi_{x}(\tau, u) e^{j 2 \pi W \tau}$ \\
\hline Duality & if $x(t) \longleftrightarrow X(u)$, then \\
\hline $\begin{array}{l}\text { Chirped } \\
\text { Signals }\end{array}$ & $\begin{array}{c}\chi_{X(t)}(\tau, u)=e^{j 2 \pi u \tau} \chi_{x(t)}(-u, \tau) \\
w(t)=x(t) e^{j \pi \alpha t^{2}} \Rightarrow e^{-j \pi \alpha \tau^{2}} \chi(\tau, u-\alpha \tau)\end{array}$ \\
\hline $\begin{array}{l}\text { Chirped } \\
\text { Spectral }\end{array}$ & $W(u) \star W(u)=X(u) \star X(u)$ \\
\hline $\begin{array}{l}\text { Siebert's } \\
\text { theorem }\end{array}$ & $\int_{\tau} \int_{u} \mid \chi\left(\tau,\left.u\right|^{2} e^{-j 2 \pi(\tau f-u t)} d \tau d u=\mid \chi\left(f,\left.t\right|^{2}\right.\right.$ \\
\hline $\begin{array}{l}\text { Siebert's } \\
\text { theorem }\end{array}$ & $\int_{\tau} \int_{u} \mid \chi\left(\tau,\left.u\right|^{2} e^{-j 2 \pi(\tau f-u t)} d \tau d u=\mid \chi\left(f,\left.t\right|^{2}\right.\right.$ \\
\hline $\begin{array}{l}\text { Moyal's } \\
\text { Identity }\end{array}$ & $\left|\chi_{x}(0,0)\right|^{2}=\int_{\tau} \int_{u} \mid \chi_{x}\left(\tau,\left.u\right|^{2} d u\right.$ \\
\hline Maxima & $\chi_{x}(0,0) \geq\left\|\chi_{x \cdot k}(\tau, u)\right\|$ \\
\hline Product & $\chi_{x \cdot k}(\tau, u)=\chi_{g}(\tau, u) \underset{u}{*} \chi_{k}(\tau, u)$ \\
\hline Convolution & $\chi_{x * h}(\tau, u)=\chi_{x}(\tau, u) \underset{\tau}{*} \chi_{h}(\tau, u)$ \\
\hline
\end{tabular}

TABLE II: Properties of the ambiguity function. Homog = Homogeneity, Corr $=$ Correlation, $\mathrm{AF}=$ Ambiguity Function . $*$
$\eta$ , means convolution with respect to $\eta$.

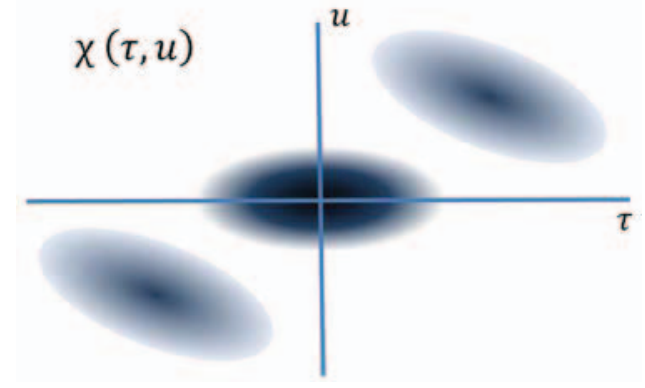

Fig. 8: Illustration of the symmetry of the ambiguity function of an arbitrary signal as described in (31) and (32).

Proof: Using the ambiguity function definition in (1),

$$
\begin{aligned}
& \int_{\tau} \int_{u} \chi(\tau, u) d \tau d u \\
= & \int_{\tau} \int_{u}\left[\int_{t} x(t) x^{*}(t-\tau) e^{-j 2 \pi u t} d t\right] d \tau d u \\
= & \int_{\tau} \int_{t} x(t) x^{*}(t-\tau)\left[\int_{u} e^{-j 2 \pi u t} d u\right] d t d \tau \\
= & \int_{\tau}\left[\int_{t} x(t) x^{*}(t-\tau) \delta(t) d t\right] d \tau \\
= & \int_{\tau} x(0) x^{*}(-\tau) d \tau \\
= & x(0) X^{*}(0)
\end{aligned}
$$

5) Ambiguity Function Symmetry:

a) General.: For an arbitrary complex signal, the ambiguity function has the following symmetry.

$$
\chi^{*}(-\tau, u)=\chi(\tau,-u) e^{-j 2 \pi u \tau}
$$

from which it follows that

$$
\left|\chi^{*}(-\tau, u)\right|=|\chi(\tau,-u)| .
$$

This symmetry is illustrated in Figure 8.

Proof: From the definition of the ambiguity function in $(1)$

$$
\chi^{*}(-\tau, u)=\int_{-\infty}^{\infty} x^{*}(\xi) x(\xi+\tau) e^{j 2 \pi u \xi} d \xi .
$$

Setting $t=\xi+\tau$ gives

$$
\begin{aligned}
\chi^{*}(-\tau, u) & =\int_{-\infty}^{\infty} x^{*}(t-\tau) x(t) e^{j 2 \pi u(t-\tau)} d t \\
& =e^{-j 2 \pi u \tau} \int_{-\infty}^{\infty} x(t) x^{*}(t-\tau) e^{j 2 \pi u t} d t
\end{aligned}
$$

which gives the desired answer in (31).

b) For Real Signals.: If $x(t)$ is real, then

$$
\chi(\tau, u)=\chi^{*}(\tau,-u) .
$$

This is the same conjugate symmetric relationship characteristic of the Fourier transform of real signals and is illustrated in Figure 9. Combined with the general symmetry illustrated 


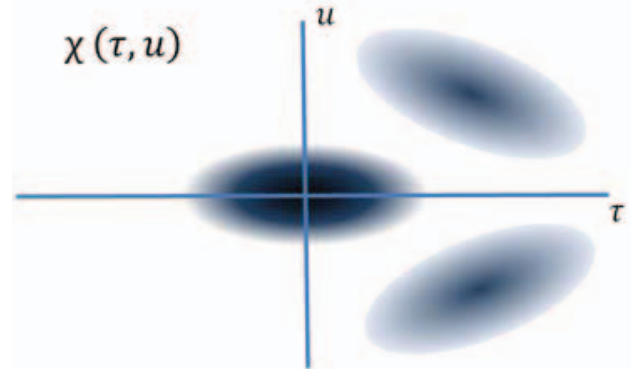

Fig. 9: Illustration of the symmetry of the ambiguity function of real signals as described in (33).

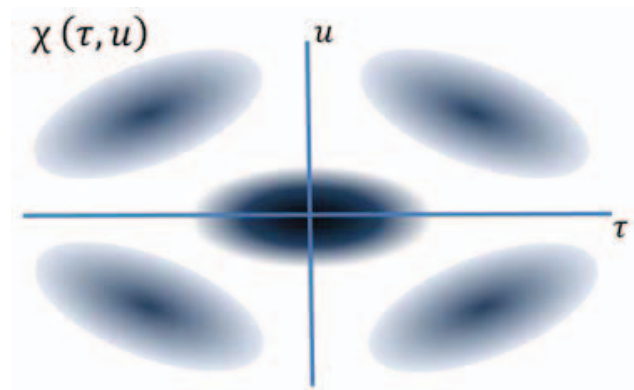

Fig. 10: Illustration of the four fold symmetry of the ambiguity function of real signals summarized in (34).

in Figure 8, real signals therefore have the four fold symmetry illustrated in Figure 10.

$$
|\chi(\tau, u)|=|\chi(\tau,-u)|=|\chi(-\tau,-u)|=|\chi(-\tau, u)|
$$

Proof: When $x(t)$ is real, the ambiguity function in (1) becomes

$$
\chi(\tau, u)=\int_{-\infty}^{\infty} x(t) x(t-\tau) e^{-j 2 \pi u t} d t .
$$

Thus

$$
\chi^{*}(\tau, u)=\int_{-\infty}^{\infty} x(t) x(t-\tau) e^{j 2 \pi u t} d t
$$

from which (33) follows.

6) Complex Heterodyned Signals: If

$$
y(t)=x(t) e^{j 2 \pi W t},
$$

then the ambiguity functions of the two signals are related by

$$
\chi_{y}(\tau, u)=\chi_{x}(\tau, u-W)
$$

Proof:

$$
\begin{aligned}
\chi_{y}(\tau, u) & =\int_{t} y(t) y^{*}(t-\tau) e^{-j 2 \pi u t} d t \\
& =\int_{t} x(t) x^{*}(t-\tau) e^{-j 2 \pi(u-W) t} d t \\
& =\chi_{x}(\tau, u-W)
\end{aligned}
$$

7) Frequency Preservation: The linear time invariant (LTI) system [24], [26], [31] has the property of preserving frequency in the sense that a complex sinusoid input of frequency $W$ outputs a complex sinusoid with the same frequency with a different amplitude and phase. Specifically, if an LTI system has impulse response $h(t)$ and $h(t) \leftrightarrow H(u)$ then the system's response to an input of $e^{j 2 \pi W t}$ is

$$
h(t) * e^{j 2 \pi W t}=H(W) e^{j 2 \pi W t}
$$

The ambiguity function has a similar property in that, if $x(t) \Rightarrow \chi_{x}(\tau, u)$, then

$$
x(t) e^{j 2 \pi W t} \Rightarrow \chi_{x}(\tau, u) e^{j 2 \pi W \tau} .
$$

Proof: By definition

$$
\begin{aligned}
x(t) e^{j 2 \pi W t} \Rightarrow & \int_{t}\left(x(t) e^{j 2 \pi W t}\right) \\
& \times\left(x^{*}(t-\tau) e^{-j 2 \pi W(t-\tau)}\right) e^{-j 2 \pi u t} d t \\
& =e^{j 2 \pi W \tau} \int_{t} x(t) x^{*}(t-\tau) e^{-j 2 \pi u t} d t
\end{aligned}
$$

which gives (35).

8) Chirped Signals: Chirping a signal is equivalent to a time dependent frequency shift in the ambiguity plane. Specifically,

$$
x(t) e^{j \pi \alpha t^{2}} \Rightarrow e^{-j \pi \alpha \tau^{2}} \chi(\tau, u-\alpha \tau)
$$

Proof:

$$
\begin{aligned}
x(t) e^{j \pi \alpha t^{2} \Rightarrow} & \int_{-\infty}^{\infty}\left[x(t) e^{j \pi \alpha t^{2}}\right] \\
& h 2 \times\left[x^{*}(t-\tau) e^{-j \pi \alpha(t-\tau)^{2}}\right] e^{-j 2 \pi u t} d t \\
& =e^{-j \pi \alpha \tau^{2}} \int_{-\infty}^{\infty} x(t) x^{*}(t-\tau) e^{-j 2 \pi t(u-\alpha \tau)} d t \\
& =e^{-j \pi \alpha \tau^{2}} \chi(\tau, u-\alpha \tau)
\end{aligned}
$$

A geometric visualization of the chirped signal theorem is in Figure 11 where, on the left, we see ${ }^{6}$

$$
\vartheta(\tau, u)=\Pi\left(\frac{\tau}{2 T}\right) \Pi\left(\frac{u}{2 B}\right)
$$

Then

$$
\vartheta(\tau, u-\alpha \tau)=\Pi\left(\frac{\tau}{2 T}\right) \Pi\left(\frac{u-\alpha \tau}{2 B}\right)
$$

is illustrated on the right hand side. The function $\Pi\left(\frac{u-\alpha \tau}{2 B}\right)$ is $\Pi\left(\frac{u}{2 B}\right)$ centered on the line $u=\alpha \tau$.

a) The Ambiguity Function of an Infinite Chirp: . The ambiguity function for $x(t)=1$, from (1), is

$$
\chi(\tau, u) \Rightarrow \int_{t} e^{-j 2 \pi u t} d t=\delta(u)
$$

6 The function in (38) and pictured in Figure 11 cannot be an ambiguity function and is used only for purposes of illustrating the functional relationship in the chirped signal theorem in (36). 


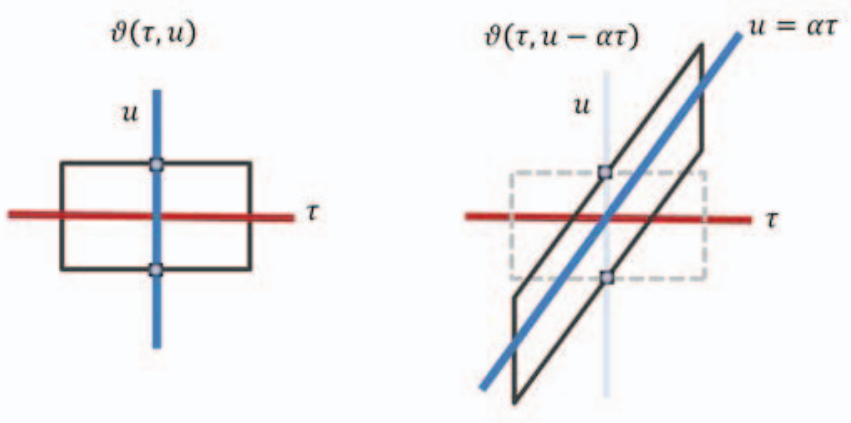

Fig. 11: Illustration of the effect on an ambiguity function by chirping. (See (37).) The rectangle in on the left from (38) has a width or $2 T$ and a height of $2 B$. The time shift in (39) is shown on the right.

From (37), it follows that the ambiguity of an infinity duration chirp is

$$
e^{j \pi \alpha t^{2}} \Rightarrow e^{-j \pi \alpha \tau^{2}} \delta(u-\alpha \tau)
$$

b) The Ambiguity Function of a Finite Chirp: . The ambiguity function for a chirp on the interval $-T \leq t \leq T$ follows from the ambiguity function of the rectangle in Table I and the chirp theorem in (37).

$$
\begin{aligned}
e^{j \pi \alpha t^{2}} \Pi\left(\frac{t}{2 T}\right) \Rightarrow & e^{-j \pi u \tau}|2 T-| \tau|| \\
& \times \operatorname{sinc}((2 T-|\tau|)(u-\alpha \tau))) \Pi\left(\frac{\tau}{4 T}\right)
\end{aligned}
$$

c) Chirped Spectral Correlation: The spectral correlation of a chirped signal is the same as the spectral correlation of the unchirped signal. If the chirped signal be

$$
w(t)=x(t) e^{j \pi \alpha t^{2}} .
$$

then

$$
W(u) \star W(u)=X(u) \star X(u) .
$$

Proof: Using the chirp ambiguity function relationship in

$$
\chi_{w}(0, u)=\chi_{x}(0, u)
$$

Using the spectral correlation in (27) immediately gives (40).

9) Duality: Fourier transform duality [31] says that if

$$
x(t) \longleftrightarrow X(u),
$$

then

$$
X(t) \longleftrightarrow x(-u) .
$$

We can use this property to find the ambiguity function of $X(t)$ if we know the ambiguity function of $x(t)$.

The ambiguity function for $X(t)$ is

$$
\chi_{X(t)}(\tau, u)=\int_{-\infty}^{\infty} X(t) X^{*}(t-\tau) e^{-j 2 \pi t u} d t
$$

and is related to $\chi_{x(t)}$ by

$$
\chi_{X(t)}(\tau, u)=e^{-j 2 \pi u \tau} \chi_{x(t)}(-u, \tau) .
$$

Proof: Reverse $\tau$ and $u$ in (41).

$$
\chi_{X(t)}(u, \tau)=\int_{-\infty}^{\infty} X(\xi) X^{*}(\xi-u) e^{-j 2 \pi \xi \tau} d \xi .
$$

Using (43) we can write (21) as

$$
\begin{aligned}
\chi_{x(t)}(\tau, u) & =e^{-j 2 \pi u \tau} \int_{-\infty}^{\infty} X(\xi) X^{*}(\xi-u) e^{j 2 \pi \xi \tau} d \xi \\
& =e^{-j 2 \pi u \tau} \chi_{X(t)}(u,-\tau)
\end{aligned}
$$

or

$$
\chi_{X(t)}(u,-\tau)=e^{j 2 \pi u \tau} \chi_{x(t)}(\tau, u) .
$$

Replacing $\tau \rightarrow-\tau$ gives

$$
\chi_{X(t)}(u, \tau)=e^{-j 2 \pi u \tau} \chi_{x(t)}(-\tau, u) .
$$

Switching $\tau$ and $u$ results in

$$
\chi_{X(t)}(\tau, u)=e^{-j 2 \pi u \tau} \chi_{x(t)}(-u, \tau)
$$

which is (42).

a) Example: Duality for a Rectangular Pulse is a Sinc Pulse: For $x(t)=\Pi\left(\frac{t}{2 \Upsilon}\right)$ in Table $\mathrm{I}^{7}$

$$
\chi_{x(t)}(\tau, u)=e^{-j \pi u \tau}|2 \Upsilon-| \tau|| \operatorname{sinc}((2 \Upsilon-|\tau|) u) \Pi\left(\frac{\tau}{4 \Upsilon}\right) .
$$

The duality zero can be applied to this result to generate the ambiguity function for a sinc.

Reversing $u$ and $\tau$,

$\chi_{x(t)}(u, \tau)=e^{-j \pi u \tau}|2 \Upsilon-| u|| \operatorname{sinc}((2 \Upsilon-|u|) \tau) \Pi\left(\frac{u}{4 \Upsilon}\right)$.

and, setting $u \rightarrow-u$,

$\chi_{x(t)}(-u, \tau)=e^{j \pi u \tau}|2 \Upsilon-| u|| \operatorname{sinc}((2 \Upsilon-|u|) \tau) \Pi\left(\frac{u}{4 \Upsilon(45)}\right)$.

Using symmetry

$\chi_{X(t)}(\tau, u)=e^{-j \pi u \tau}|2 \Upsilon-| u|| \operatorname{sinc}((2 \Upsilon-|u|) \tau) \Pi\left(\frac{u}{4 \Upsilon}\right)$.

If we multiply (45) by $e^{-j 2 \pi u \tau}$, we obtain (46) thereby verifying the duality theorem in (42) and the corresponding entry in Table I for the sinc function.

10) Product :

$$
\chi_{g \cdot k}(\tau, u)=\chi_{g}(\tau, u) \underset{u}{*} \chi_{k}(\tau, u) .
$$

In other words, if

$$
x(t)=g(t) k(t),
$$

then

$$
\chi_{x}(\tau, u)=\chi_{g}(\tau, u) \underset{u}{*} \chi_{k}(\tau, u) .
$$

\footnotetext{
${ }^{7}$ We will use $\Upsilon$ for both $T$ and $B$ to avoid comparative confusion.
} 
Proof:

$$
\begin{aligned}
\chi_{g \cdot k}(\tau . u) & =\int_{t} g(t) k(t) g^{*}(t-\tau) k^{*}(t-\tau) e^{-j 2 \pi u t} d t \\
& =\int_{t} p(t) q^{*}(t) d t
\end{aligned}
$$

where

$$
p(t)=g(t) g^{*}(t-\tau)
$$

and

$$
q(t)=k^{*}(t) k(t-\tau) e^{j 2 \pi u t} .
$$

Applying the power theorem [31] to (48).

$$
\chi_{g \cdot k}(\tau, u)=\int_{f} P(f) Q^{*}(f) d f .
$$

Since

$$
\begin{aligned}
P(f) & =\int_{t} g(t) g^{*}(t-\tau) e^{-j 2 \pi f t} d t \\
& =\chi_{g}(\tau, f)
\end{aligned}
$$

and

$$
\begin{aligned}
Q(f) & =\int_{t} k^{*}(t) k(t-\tau) e^{-j 2 \pi(f-u) t} d t \\
& =\left[\int_{t} k(t) k^{*}(t-\tau) e^{-j 2 \pi(u-f) t} d t\right]^{*} d t \\
& =\chi_{k}^{*}(\tau, u-f) .
\end{aligned}
$$

Substituting (50) and (51) into (49) gives

$$
\chi_{g \cdot k}(\tau, u)=\int_{f} \chi_{g}(\tau, f) \chi_{k}(\tau, u-f) d f
$$

which can be written as (47).

11) Convolution: Let $x(t)$ be passed through a filter with impulse response $h(t)$. The filter output is

$$
z(t)=x(t) * h(t) .
$$

Then

$$
\chi_{z}(\tau, u)=\chi_{x}(\tau, u) \underset{\tau}{*} \chi_{h}(\tau, u)
$$

or

$$
\chi_{x * h}(\tau, u)=\chi_{x}(\tau, u) \underset{\tau}{*} \chi_{h}(\tau, u) .
$$

This is proved in Appendix VII-B1

12) Siebert's Theorem: Siebert's theorem [41] shows that the two dimensional Fourier transform of the squared magnitude of the ambiguity function is the same function rotated $90^{\circ}$. Specifically

$$
\int_{\tau} \int_{u}|\chi(\tau, u)|^{2} e^{-j 2 \pi(\tau f-u t)} d \tau d u=|\chi(f, t)|^{2}
$$

Proof: From Figure 13 and (66), we can discern the 2D Fourier transform of the ambiguity function.

$$
\begin{aligned}
\int_{\tau} \int_{u} \chi(\tau, u) e^{-j 2 \pi(\tau f-u t)} d \tau d u & =\Psi(f, t) \\
& =x(t) X^{*}(f) e^{-j 2 \pi f t}
\end{aligned}
$$

Therefore the integral in (55) is a two dimensional autocorrelation

$$
\begin{aligned}
& \int_{\tau} \int_{u}\left|\chi_{x}(\tau, u)\right|^{2} e^{-j 2 \pi(\tau f-u t)} d \tau d u \\
= & \Psi(f, t) \star \Psi(f, t) \\
= & \int_{\tau} \int_{u}\left[x(\tau) X^{*}(u) e^{-j 2 \pi u \tau}\right] \\
& \times\left[x(\tau-t) X^{*}(u-f) e^{-j 2 \pi(u-f)(\tau-t)]^{*}} d u d \tau\right. \\
= & \int_{\tau} \int_{u}\left[x(\tau) X^{*}(u) e^{-j 2 \pi u \tau}\right] \\
& \times\left[x^{*}(\tau-t) X(u-f) e^{j 2 \pi(u-f)(\tau-t)}\right] d u d \tau \\
= & \int_{u}\left\{\left[\int_{\tau} x(\tau) x^{*}(\tau-t) e^{-j 2 \pi u \tau} e^{j 2 \pi(u-f) \tau} d \tau\right]\right. \\
& \left.\times X^{*}(u) X(u-f) e^{-j 2 \pi(u-f) t}\right\} d u \\
= & \int_{\tau} x(\tau) x^{*}(\tau-t) e^{-j 2 \pi f \tau} d \tau \\
& \times \int_{u} X^{*}(u) X(u-f) e^{-j 2 \pi(u-f)} d u \\
= & \chi_{x}(f, t)\left[e^{-j 2 \pi f} \int_{u} X(u) X^{*}(u-f) e^{j 2 \pi u} d u\right]^{*}
\end{aligned}
$$

Using the ambiguity expression in (21) gives

$$
\int_{\tau} \int_{u} \mid \chi_{x}\left(\tau,\left.u\right|^{2} e^{-j 2 \pi(\tau f-u t)} d \tau d u=\chi_{x}(f, t) \chi_{x}^{*}(f, t)\right.
$$

which is Siebert's Theorem in (55).

a) Moyal's Identity: [15] The area of the magnitude squared of the ambiguity function is equal to the magnitude squared of the origin.

$$
\left|\chi_{x}(0,0)\right|^{2}=\int_{\tau} \int_{u} \mid \chi_{x}\left(\tau,\left.u\right|^{2} d u\right.
$$

This follows immediately from Siebert's theorem in (56) for $f=t=0$.

$$
\int_{\tau} \int_{u}|\chi(\tau . u)|^{2} d \tau d u
$$

\section{Ambiguity Functions of Narrowband Signals}

The ambiguity function of a baseband signal differs from the ambiguity of the signal modulated by an RF signal. Consider again the RF signal in (11)

$$
s_{W}(t)=\Re\left[g(t) e^{j 2 \pi W t}\right]
$$

where

$$
\begin{aligned}
g(t) & =|g(t)| e^{j \angle g(t)} \\
& =i(t)+j q(t) .
\end{aligned}
$$

$g(t)$ is a complex baseband signal with bandwidth $B$. Then $s_{W}(t)$ is a narrowband signal with carrier frequency $W>B$. The real part of $g(t)$ is $i(t)$ and is referred to as the in phase 


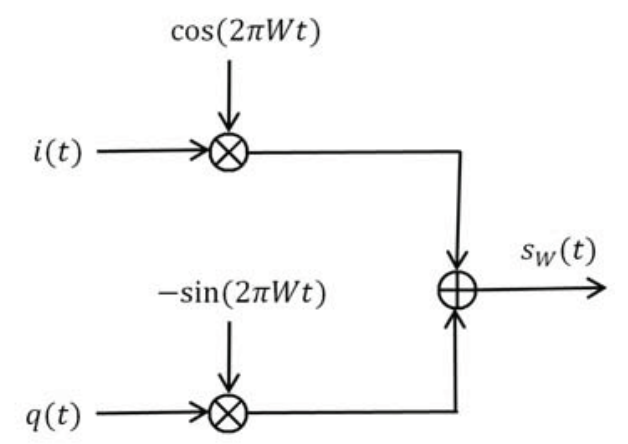

Fig. 12: Generation of the narrowband signal in (60). The sin and cos can be generated by a single oscillator using a $45^{\circ}$ phase shift.

component while the imaginary part, $q(t)$, is the quadrature signal.

\section{A. Generating the Narrowband Signal}

Substituting (59) in (57) gives

$$
\begin{aligned}
s_{W}(t) & =\Re(i(t)+j q(t))(\cos (2 \pi W t)+j \sin (2 \pi W t)) \\
& =i(t) \cos (2 \pi W t)-q(t) \sin (2 \pi W t)
\end{aligned}
$$

Generation of $s_{W}(t)$ is shown in Figure 12.

\section{B. Baseband vs. Narrowband Ambiguity}

Since the narrowband signal $s_{W}(t)$ is uniquely defined by the baseband signal, $g(t)$, it makes sense that the ambiguity function of $s_{W}$ should be able to expressed in terms of the ambiguity function of $g$. The relationship of the ambiguity functions of $g(t)$ and $s_{W}(t)$ is

$$
\begin{aligned}
\chi_{s_{w}}(\tau, u)= & \frac{1}{4}\left[e^{j 2 \pi W \tau} \chi_{g}(\tau, u)+e^{-j 2 \pi W \tau} \chi_{g}^{*}(\tau,-u)\right] \\
+ & \frac{1}{4}\left[e^{-j 2 \pi W \tau} \psi_{g}(\tau, u+2 W)\right. \\
& \left.+e^{j 2 \pi W \tau} \psi_{g}^{*}(\tau,-(u+2 W))\right]
\end{aligned}
$$

where

$$
\psi_{g}(\tau, u)=\int_{t} g(t) g(t-\tau) e^{-j 2 \pi u t} d t
$$

The $\psi_{g}$ terms are sufficiently shifted on the frequency axis to be of little or no concern. For a proof of (62), see Appendix VII-B2.

\section{2D FOURIER ANALYSIS}

The ambiguity function is a two dimensional function and is therefore subject to 2D Fourier analysis. The Fourier timefrequency variable pairs are $(\tau, f)$ and $(t, u)$ and we adopt the notation

$$
\begin{aligned}
\mathcal{F}_{\tau \rightarrow f} \chi(\tau, u) & :=\Lambda(f, u) \\
& =\int_{-\infty}^{\infty} \chi(\tau, u) e^{-j 2 \pi \tau f} d \tau
\end{aligned}
$$

Here are the mappings between all of the possible frequency and time variables.

$$
\begin{array}{rll}
\chi(\tau, u) & \stackrel{\tau \rightarrow f}{\longrightarrow} & \Gamma(f, u) \\
t \rightarrow u \quad \uparrow & 2 D \nearrow & \uparrow \quad t \rightarrow u \\
\Lambda(\tau, t) & \stackrel{ }{\tau \rightarrow f} & \Psi(f, t)
\end{array}
$$

These Fourier mappings are pictured in Figure 13 in more detail. The ambiguity function is on the top left in Figure 13. The arrows denote the direction of a one-dimensional Fourier transform along either rows or columns.

1) On the $(\tau, u)$ plane: Using (20), the projection [31], [34] of $\chi(\tau, u)$ on $u$ is

$$
\begin{aligned}
\int_{\tau} \chi_{x}(\tau, u) d \tau & =\int_{\beta} X(\beta+u) X^{*}(\beta)\left[\int_{\tau} e^{j 2 \pi \beta \tau} d \tau\right] d \beta \\
& =\int_{\beta} X(\beta+u) X^{*}(\beta) \delta(\beta) d \beta \\
& =X(u) X^{*}(0)
\end{aligned}
$$

Likewise, on $\tau$,

$$
\begin{aligned}
\int_{u} \chi(\tau, u) d u & =\int_{\beta}\left[\int_{u} X(\beta+u) d u\right] X^{*}(\beta) e^{j 2 \pi \beta \tau} d \beta \\
& =x(0)\left[\int_{\beta} X(\beta) e^{-j 2 \pi \beta \tau}\right]^{*} d \beta \\
& =x(0) x^{*}(-\tau)
\end{aligned}
$$

The axes and projection properties are illustrated in the upper left figure in Figure 13.

We conclude from (64) and (65) that, to within a proportionality constant, the ambiguity function can be inverted to the single or spectrum that gave rise to the ambiguity function.

2) On the $(f, u)$ plane: The expression in (63) is the transform of the top left function to the top right in Figure 13. We can show that $\Lambda(f, u)$ is simply the product of two functions.

$$
\Lambda(f, u)=X^{*}(f) X(u+f) .
$$

Proof: The ambiguity function in (20) is recognized as the inverse Fourier transform of $\Lambda(f, u)$.

The value along the $u$ and $f$ axes are $X^{*}(0) X(u)$ and $|X(f)|^{2}$ as shown in Figure 13. Note that the projection on $u$ of $\Lambda(f, u)$ is

$$
\begin{aligned}
\int_{f=-\infty}^{\infty} \Lambda(f, u) d u & =\int_{f=-\infty}^{\infty} X^{*}(f) X(u+f) d f \\
& =\int_{\xi=-\infty}^{\infty} X(\xi) X^{*}(\xi-u) \\
& =X(u) \star X(u)
\end{aligned}
$$

This is shown in the upper right in Figure 13. 


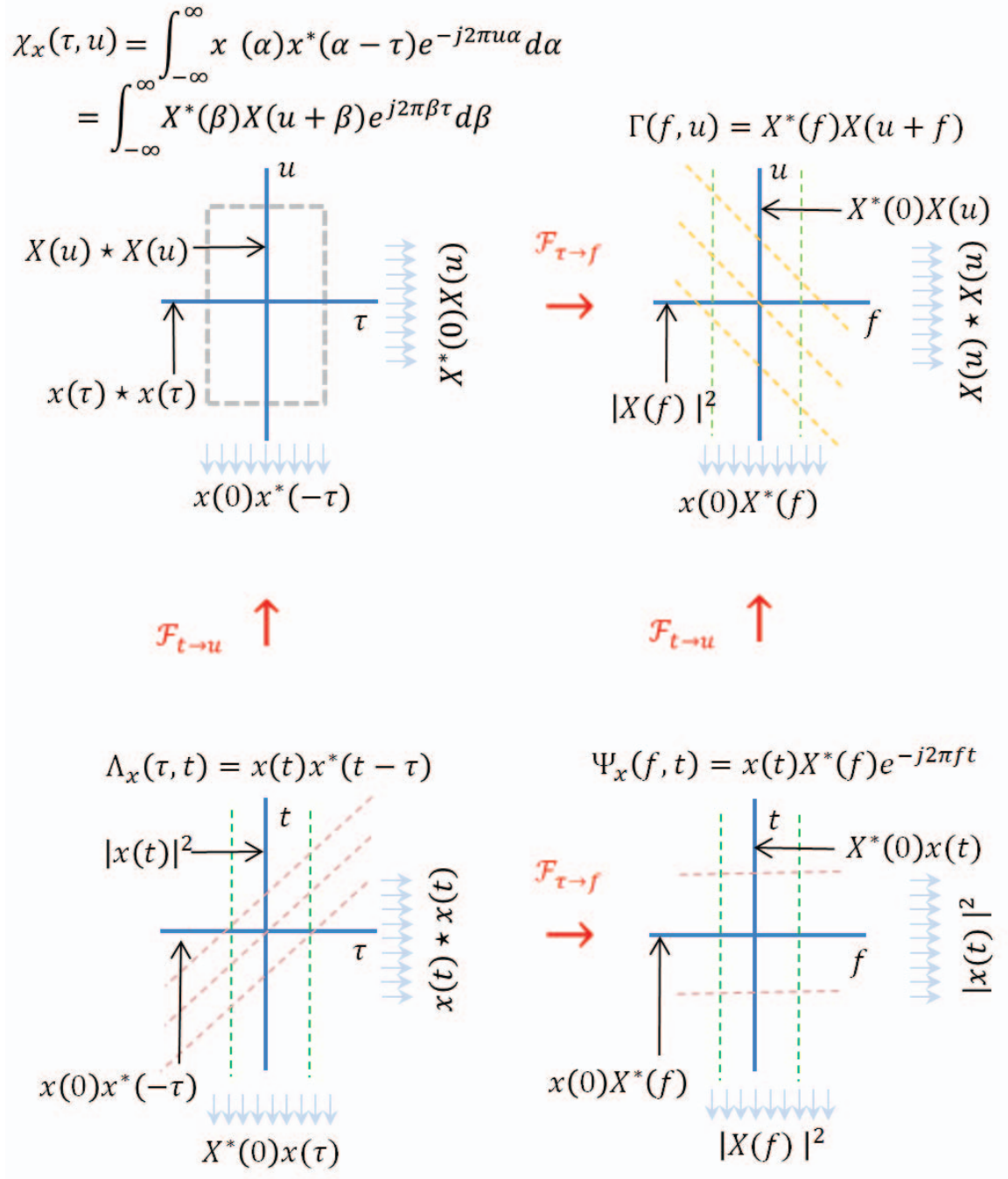

Fig. 13: 2D Fourier Analysis.

Likewise, the projection on $f$ is

$$
\begin{aligned}
\int_{u=-\infty}^{\infty} \Lambda(f, u) d u & =X^{*}(f) \int_{u=-\infty}^{\infty} X(u+f) d u \\
& =X^{*}(f) \int_{u=-\infty}^{\infty} X(u) d u \\
& =x(0) X^{*}(f)
\end{aligned}
$$

This projection is also shown in the upper right in Figure 13.

3) On the $(f, t)$ plane: Define, as shown on the bottom right of Figure 13,

$$
\Psi_{x}(f, t):=x(t) X^{*}(f) e^{-j 2 \pi f t},
$$

We conclude

$$
\begin{aligned}
\mathcal{F}_{t \rightarrow u} \Psi_{x}(f, t) & =X^{*}(f) X(u+f) \\
& =\Lambda(f, u)
\end{aligned}
$$

The values on the axes as shown in the bottom right figure of Figure 13 are $X^{*}(0) x(t)$ and $x(0) X^{*}(f)$. The projection on $t$ is

$$
\begin{aligned}
\int_{f} \Psi_{x}(f, t) d f & =x(t) \int_{f} X^{*}(f) e^{-j 2 \pi f t} d f \\
& =x(t)\left[\int_{f} X(f) e^{j 2 \pi f t} d f\right]^{*} \\
& =x(t) x^{*}(t) \\
& =|x(t)|^{2}
\end{aligned}
$$

Since

$$
\begin{aligned}
\mathcal{F}_{t \rightarrow u} x(t) e^{-j 2 \pi u t} & =\int_{t=-\infty}^{\infty} x(t) e^{-j 2 \pi(u+f) t} d t \\
& =X(u+f) .
\end{aligned}
$$


and the projection on $f$ is

$$
\begin{aligned}
\int_{t} \Psi_{x}(f, t) d t & =X^{*}(f) \int_{t} x(t) e^{-j 2 \pi f t} d t \\
& =X^{*}(f) X(f) \\
& =|X(f)|^{2}
\end{aligned}
$$

These projections are shown on the lower right in Figure 13.

4) On the $(\tau, t)$ plane: The ambiguity function is recognized as the Fourier transform in $t$ of

$$
\Lambda(\tau, t)=x(t) x^{*}(t-\tau) .
$$

That is

$$
\mathcal{F}_{t \rightarrow u} \Lambda(\tau, t)=\chi_{x}(\tau, u) .
$$

As is shown on the lower left of the figure in Figure 13, the axes values are $|x(t)|^{2}$ and $x(0) x^{*}(-\tau)$. The projection on $t$ is

$$
\int_{t} \Lambda(\tau, t) d t=x(t) \star x(t)
$$

and on $\tau$, we have

$$
\begin{aligned}
\int_{\tau} \Lambda(\tau, t) d \tau & =x(t) \int_{\tau} x^{*}(t-\tau) d \tau \\
& =x(t) \int_{\tau} x^{*}(-\tau) d \tau \\
& =x(t)\left[\int_{\xi} x(\xi) d \xi\right]^{*} \\
& =x(t) x^{*}(0)
\end{aligned}
$$

These properties are shown on the lower left figure in Figure 13.

\section{APPENDIX}

\section{A. Derivation Details}

1) Equation 13: From (11), the squared norm of $s_{W}(t)$ is

$$
\begin{aligned}
\left\|s_{W}(t)\right\|^{2} & =\int_{t=-\infty}^{\infty}\left|s_{W}(t)\right|^{2} d t \\
& =\int_{t=-\infty}^{\infty}|g(t)|^{2} \cos ^{2}(2 \pi W t+\angle g(t)) d t
\end{aligned}
$$

Using a trig identity

$$
\begin{aligned}
\left\|s_{W}(t)\right\|^{2}= & \frac{1}{2} \int_{t=-\infty}^{\infty}|g(t)|^{2} \\
& \times[1+\cos (4 \pi W t+2 \angle g(t))] d t \\
= & \frac{1}{2}\|g(t)\|^{2}+\frac{1}{2} \int_{t=-\infty}^{\infty}|g(t)|^{2} \\
& \quad \times \cos (4 \pi W t+2 \angle g(t)) d t \\
= & \frac{1}{2}\|g(t)\|^{2} \\
& \quad+\frac{1}{2} \Re \int_{t=-\infty}^{\infty}|g(t)|^{2} e^{j(4 \pi W t)+2 \angle g(t))} d t \\
= & \frac{1}{2}\|g(t)\|^{2} \\
= & +\frac{1}{2} \Re \int_{t=-\infty}^{\infty} g^{2}(t) e^{j 4 \pi W t} d t
\end{aligned}
$$

From the modulation theorem of Fourier analysis [31],

$$
g^{2}(t) \longleftrightarrow \int_{t=-\infty}^{\infty} g^{2}(t) e^{j 2 \pi u t} d t=G(u) * G(u)
$$

we conclude

$$
\int_{t=-\infty}^{\infty} g^{2}(t) e^{j 2 \pi(2 W) t} d t=\left.[G(u) * G(u)]\right|_{u=2 W} .
$$

Since $g(t)$ has bandwidth $B$, we see that $G(u) * G(u)=0$ for $|u|>2 B$. From (12), since $2 W>2 B$, (68) is therefore zero and (67) becomes (13), i.e.

$$
\left\|s_{W}(t)\right\|^{2}=\frac{1}{2}\|g(t)\|^{2}
$$

2) Equation 16: To prove the matched filter result in (16), we wish to find

$$
\begin{aligned}
& \min _{\tau, \nu}\left\|s_{W+\Delta W}(t-\Delta t)-s_{W+\nu}(t-\tau)\right\|^{2} \\
= & \min _{\tau, \nu}\left[\left\|s_{W+\Delta W}(t-\Delta t)\right\|^{2}+\left\|s_{W+\nu}(t-\tau)\right\|^{2}\right. \\
& \left.-2 \Re \int_{t=-\infty}^{\infty} s_{W+\Delta W}(t-\Delta t) s_{W+\nu}^{*}(t-\tau) d t\right]
\end{aligned}
$$

From (13),

$$
\left\|s_{W+\Delta W}(t-\Delta t)\right\|^{2}=\left\|s_{W+\nu}(t-\tau)\right\|^{2}=\frac{1}{2}\|g(t)\|^{2},
$$

so that

$$
\begin{gathered}
\min _{\tau, \nu}\left\|s_{W+\Delta W}(t-\Delta t)-s_{W+\nu}(t-\tau)\right\|^{2} \\
=\min _{\tau, \nu}\left[\|g(t)\|^{2}-2 \Re\left\langle s_{W+\Delta W}(t-\Delta t) \mid s_{W+\nu}(t-\tau)\right\rangle\right] .
\end{gathered}
$$

The quantity $\|g(t)\|^{2}$ is not a function of either $\tau$ or $\nu$ and therefore has no effect on the maximization. The minimization in (69) is therefore equivalent to maximization of

$$
\Re \int_{-\infty}^{\infty} s_{W+\Delta W}(t-\Delta t) s_{W+\nu}^{*}(t-\tau) d t
$$

This correlation can be expressed using the ambiguity function. Since $z+z^{*}=2 \Re z$, (11) can be written as the conjugate sum

$$
s_{W}(t)=\frac{1}{2} g(t) e^{j 2 \pi W t}+\frac{1}{2} g^{*}(t) e^{-j 2 \pi W t}
$$

and

$$
\begin{gathered}
\Re \int_{-\infty}^{\infty} s_{W+\Delta W}(t-\Delta t) s_{W+\nu}^{*}(t-\tau) d t \\
=\frac{1}{4} \Re \int_{-\infty}^{\infty} s_{W+\Delta W}(t-\Delta t) s_{W+\nu}^{*}(t-\tau) d t \\
=\frac{1}{4} \int_{-\infty}^{\infty}\left[g(t-\Delta t) e^{j 2 \pi(W+\Delta W)(t-\Delta t)}\right. \\
\left.\quad+g^{*}(t-\Delta t) e^{-j 2 \pi(W+\Delta W)(t-\Delta t)}\right] \\
\times\left[g^{*}(t-\tau) e^{-j 2 \pi(W+\nu)(t-\tau)}\right. \\
\left.+g(t-\tau) e^{j 2 \pi(W+\nu)(t-\tau)}\right] d t
\end{gathered}
$$




$$
\begin{aligned}
=\frac{1}{4} & \int_{-\infty}^{\infty} g(t-\Delta t) g^{*}(t-\tau) e^{j 2 \pi(W+\Delta W)(t-\Delta t)} \\
& \times e^{-j 2 \pi(W+\nu)(t-\tau)} d t \\
+ & \frac{1}{4} \int_{-\infty}^{\infty} g^{*}(t-\Delta t) g(t-\tau) e^{-j 2 \pi(W+\Delta W)(t-\Delta t)} \\
& \times e^{j 2 \pi(W+\nu)(t-\tau)} d t \\
+ & \frac{1}{4} \int_{-\infty}^{\infty} g(t-\Delta t) g(t-\tau) e^{j 2 \pi(W+\Delta W)(t-\Delta t)} \\
& \times e^{j 2 \pi(W+\nu)(t-\tau)} d t \\
+ & \frac{1}{4} \int_{-\infty}^{\infty} g^{*}(t-\Delta t) g^{*}(t-\tau) e^{-j 2 \pi(W+\Delta W)(t-\Delta t)} \\
& \times e^{-j 2 \pi(W+\nu)(t-\tau)} d t
\end{aligned}
$$

In the above four terms, the first two are complex conjugate pairs as are the last two. Thus

$$
\begin{aligned}
\Re & \left\langle s_{W+\Delta W}(t-\Delta t) \mid s_{W+\nu}(t-\tau)(t-\tau)\right\rangle \\
= & \Re\left[\left(e^{-j 2 \pi(W+\Delta W) \Delta t} e^{j 2 \pi(W+\nu) \tau}\right.\right. \\
& \left.\times \int_{-\infty}^{\infty} g(t-\Delta t) g^{*}(t-\tau) e^{-j 2 \pi((W+\nu)-(W+\Delta W)) t} d t\right) \\
+ & \left(e^{-j 2 \pi(W+\Delta W) \Delta t} e^{-j 2 \pi u \tau}\right. \\
& \left.\left.\times \int_{-\infty}^{\infty} g(t-\Delta t) g(t-\tau) e^{j 2 \pi((W+\nu)+(W+\Delta W)) t} d t\right)\right] \\
2 \Re & e^{-j 2 \pi(W+\Delta W) \Delta t}\left[\left(e^{j 2 \pi(W+\nu) \tau}\right.\right. \\
\times & \left.\int_{-\infty}^{\infty} g(t-\Delta t) g^{*}(t-\tau) e^{-j 2 \pi(\nu-\Delta W)) t} d t\right) \\
+ & \left(e^{-j 2 \pi(\nu-\Delta W) \tau}\right. \\
& \left.\left.\times \int_{-\infty}^{\infty} g(t-\Delta t) g(t-\tau) e^{j 2 \pi(2 W+\nu+\Delta W)) t} d t\right)\right]
\end{aligned}
$$
is

1) Equation 54 : The ambiguity function for $z(t)$ in (52)

$$
\begin{aligned}
\chi_{z}(\tau, u)= & \int_{t} z(t) z^{*}(t-\tau) e^{-j 2 \pi u t} d t \\
= & \int_{t}\left\{\left[\int_{\alpha} x(\alpha)\right) h(t-\alpha) d \alpha\right] \\
& {\left.\left[\int_{\beta} x^{*}(\beta) h^{*}(t-\beta-\tau) d \beta\right] e^{-j 2 \pi u t}\right\} d t } \\
= & \int_{\alpha} \int_{\beta} h(\alpha) h^{*}(\beta)\left[\int_{t} x(t-\alpha) x^{*}(t-\tau-\beta)\right. \\
& \left.\times e^{-j 2 \pi u t} d t\right] d \alpha d \beta
\end{aligned}
$$

Set $\xi=t-\alpha$ so that $t=\xi+\alpha$. Then

$$
\begin{aligned}
\chi_{z}(\tau, u)= & \int_{\alpha} \int_{\beta} h(\alpha) h^{*}(\beta)\left[\int_{\xi} x(\xi) x^{*}(\xi+\alpha-\tau-\beta)\right. \\
& \left.\times e^{-j 2 \pi u(\xi+\alpha)} d \xi\right] d \alpha d \beta \\
= & \int_{\alpha} \int_{\beta} h(\alpha) h^{*}(\beta) \\
& \times\left[\chi_{x}(\tau-(\alpha-\beta), u) e^{-j 2 \pi u \alpha}\right] d \alpha d \beta
\end{aligned}
$$

Set $\gamma=\alpha-\beta$ so that $\beta=\alpha-\gamma$.

$$
\begin{aligned}
\chi_{z}(\tau, u)= & \int_{\alpha} \int_{\gamma} h(\alpha) h^{*}(\alpha-\gamma) \chi_{x}(\tau-\gamma, u) \\
& \times e^{-j 2 \pi u \alpha} d \alpha d \gamma \\
= & \left.\int_{\gamma}\left[\int_{\alpha} h(\alpha) h^{*} \alpha-\gamma\right) e^{-j 2 \pi u \alpha} d \alpha\right] \\
& \times \chi_{x}(\tau-\gamma, u) d \gamma \\
= & \int_{\gamma} \chi_{h}(\gamma, u) \chi_{x}(\tau-\gamma, u) d \gamma \\
= & \chi_{h}(\tau, u) * \chi_{\tau}(\tau, u)
\end{aligned}
$$

which is the desired result in (53).

2) Equation 61: To prove the relationship between narrow band and baseband ambiguity function in (61), first consider

$$
\begin{aligned}
\chi_{s_{w}}(\tau, u)= & \int_{t} s_{W}(t) s_{W}(t-\tau) e^{-j 2 \pi u t} d t \\
= & \int_{t}\left(\Re g(t) e^{j 2 \pi W t}\right) \Re(g(t-\tau) \\
& \left.\times e^{j 2 \pi W(t-\tau)}\right) e^{-j 2 \pi u t} d t
\end{aligned}
$$

Since $\Re z=\frac{1}{2}\left(z+z^{*}\right)$, 


$$
\begin{aligned}
& \chi_{s_{w}}(\tau, u) \\
& =\frac{1}{4} \int_{t}\left(g(t) e^{j 2 \pi W t}+g^{*}(t) e^{-j 2 \pi W t}\right) \\
& \times\left(g(t-\tau) e^{j 2 \pi W(t-\tau)}+g^{*}(t-\tau) e^{-j 2 \pi W(t-\tau)}\right) \\
& \times e^{-j 2 \pi u t} d t \\
& =\frac{1}{4} \int_{t}\left[g(t) e^{j 2 \pi W t} g^{*}(t-\tau) e^{-j 2 \pi W(t-\tau)} d t\right. \\
& +g^{*}(t) e^{-j 2 \pi W t} g(t-\tau) e^{j 2 \pi W(t-\tau)} \\
& +g(t) e^{j 2 \pi W t} g(t-\tau) e^{j 2 \pi W(t-\tau)} \\
& \left.+g^{*}(t) e^{-j 2 \pi W t} g^{*}(t-\tau) e^{-j 2 \pi W(t-\tau)}\right] e^{-j 2 \pi u t} d t \\
& =\frac{1}{4}\left[e^{j 2 \pi W \tau} \int_{t} g(t) g^{*}(t-\tau) e^{-j 2 \pi u t} d t\right. \\
& +e^{-j 2 \pi W \tau} \int_{t} g^{*}(t) g(t-\tau) e^{-j 2 \pi u t} d t \\
& +e^{-j 2 \pi W \tau} \int_{t} g(t) g(t-\tau) e^{-j 2 \pi(u-2 W) t} d t \\
& \left.+e^{j 2 \pi W \tau}\left(\int_{t} g(t) g(t-\tau) e^{j 2 \pi(u+2 W) t} d t\right)^{*}\right] \\
& =\frac{1}{4}\left[e^{j 2 \pi W \tau} \chi_{g}(\tau, u)+e^{-j 2 \pi W \tau} \chi_{g}^{*}(\tau,-u)\right] \\
& +\frac{1}{4}\left[e^{-j 2 \pi W \tau} \psi_{g}(\tau, u+2 W)\right. \\
& \left.+e^{j 2 \pi W \tau} \psi_{g}^{*}(\tau,-(u+2 W))\right]
\end{aligned}
$$

where $\psi_{g}(\tau, u)$ is given in (62).

\section{REFERENCES}

[1] Luis B. Almeida, "The fractional Fourier transform and time-frequency representations. Signal Processing, IEEE Transactions on 42, no. 11 (1994): 3084-3091

[2] H. Bartelt, J. Ojeda-Castañeda, and Enrique E. Sicre. "Misfocus tolerance seen by simple inspection of the ambiguity function." Applied optics 23, no. 16 (1984): 2693-2696.

[3] Charles Baylis, Robert J. Marks II, "Frequency multiplexing tickle tones to determine harmonic coupling weights in nonlinear systems," Microwave Measurement Symposium (ARFTG). Tempe, Arizona. 2011 78th ARFTG pp.1-7, 1-2 Dec. 2011.

[4] Charles Baylis II and Robert J. Marks II, "Small Perturbation Harmonic Coupling In Nonlinear Periodicity Preservation Systems," IEEE Transactions on Circuits and Systems I: Regular Papers, vol.59, no.12, pp.30343045, Dec. 2012.

[5] Charles Baylis II and Robert J. Marks II, "Evaluation of Harmonic Coupling Weights in Nonlinear Periodicity Preservation Systems," IEEE Transactions on Circuits and Systems I: Regular Papers, vol.59, no.12, pp.3024-3033, Dec. 2012.

[6] R. H. Barker, "Group synchronizing of binary digital systems." Communication Theory (1953): 273-287.

[7] Brenner, K-H., A. W. Lohmann, and Jorge Ojeda-Castaeda. "The ambiguity function as a polar display of the OTF." Optics Communications 44, no. 5 (1983): 323-326.

[8] Cheney, Margaret. "A mathematical tutorial on synthetic aperture radar." SIAM review 43, no. 2 (2001): 301-312

[9] K.F. Cheung, L.E. Atlas, J.A. Ritcey, C.A. Green and R.J. Marks II, "Conventional and composite matched filters with error correction: a comparison," Applied Optics, vol. 26, pp.4235-4239 (1987).

[10] K.F. Cheung and R.J. Marks II, "Image sampling below the Nyquist density without aliasing," Journal of the Optical Society of America A, vol.7, pp.92-105 (1990).
[11] John C. Curlanderand and Robert N. McDonough. Synthetic Aperture Radar, John Wiley \& Sons, 1991.

[12] A.K. Das, R.J. Marks II, M.A. El-Sharkawi, Payman Arabshahi and Andrew Gray, "r -shrink: A Heuristic for Improving Minimum Power Broadcast Trees in Wireless Networks," IEEE Global Telecommunications Conference 2003. GLOBECOM '03. ,Volume: 1, 1-5 Dec. 2003, pp.523 - 527.

[13] H. J. Einhorn \& R. M. Hogarth, "Decision making under ambiguity: A note," (pp. 327-336). Springer Netherlands. (1988).

[14] Dylan Eustice, Charles Baylis, Lawrence Cohen, Robert Marks "Mythbusting: Exploring Common Misconceptions about the Ambiguity Function, 2015 IEEE International Symposium on Antennas and Propagation and North American Radio Science Meeting in Vancouver, British Columbia, Canada, July 19-25, 2015.

[15] David C. Farden and Louis L. Scharf. "A unified framework for the Sussman, Moyal, and Janssen formulas." Signal Processing Magazine, IEEE 23, no. 3 (2006): 124-125.

[16] Matthew Fellows, Charles Baylis, Lawrence Cohen, and Robert J. Marks II. "Calculation of the Radar Ambiguity Function from Time-Domain Measurement Data for Real-Time, Amplifier-in-the-Loop Waveform Optimization," 2013 82nd ARFTG Microwave Measurement Conference, Ohio State University, Columbus, Ohio pp. 1-5. IEEE, 2013.

[17] Shaowei Han and Chris Rizos. "Improving the computational efficiency of the ambiguity function algorithm." Journal of Geodesy 70, no. 6 (1996): 330-341.

[18] Harold Szu and J. A. Blodgett. "Wigner distribution and ambiguity function." In Optics in Four Dimensions, vol. 65, no. 1, pp. 355-381. AIP Publishing, 1980.

[19] Amit Kedia, "A computationally efficient method for estimating the channel impulse response for the IEEE 802.11 b (WLAN)." In Communications, Computers and signal Processing, 2005. PACRIM. 2005 IEEE Pacific Rim Conference on, pp. 578-581. IEEE, 2005.

[20] B.V.K. Vijaya Kumar, Abhijit Mahalanobis, and Richard D. Juday, Correlation pattern recognition, Vol. 27. Cambridge: Cambridge University Press, 2005

[21] Philippe Lacomme, Jean-Claude Marchais, Jean-Philippe Hardange, and Eric Normant. Air and spaceborne radar systems: An introduction. William Andrew, 2001.

[22] Ka Hin Leung and Bernhard Schmidt. "The field descent method," Designs, Codes and Cryptography 36, no. 2 (2005): 171-188

[23] Nadav Levanon, Radar principles, New York, Wiley-Interscience, 1988

[24] R.J. Marks II, John F. Walkup, and Marion O. Hagler. ”A sampling theorem for space-variant systems." JOSA 66.9 (1976): 918-921.

[25] R.J. Marks II, J.F. Walkup and M.O. Hagler. "Ambiguity function display: an improved coherent processor." Applied Optics, vol. 16, pp.746-750 (1977)

[26] R.J. Marks II, John F. Walkup, Marion O. Hagler, and Thomas F. Krile. "Space-variant processing of 1-D signals." Applied optics 16, no. 3 (1977): 739-745.

[27] R.J. Marks II and M.W. Hall. "Ambiguity function display using a single one-dimensional input." Applied Optics, vol. 18, pp.2539-2540 (1979).

[28] R.J. Marks II and L.E. Atlas, "Composite matched filtering with error correction," Optics Letters, vol. 12, pp.135-137 (1987).

[29] R.J. Marks II, J.A. Ritcey, L.E. Atlas and K.F. Cheung, "Composite matched filter output partitioning," Applied Optics, vol. 26, pp.22742278 (1987).

[30] R.J. Marks II, Introduction to Shannon Sampling and Interpolation Theory, (Springer-Verlag, 1991) Softcover reprint 2012.

[31] R.J. Marks II, Handbook of Fourier analysis \& its applications. London: Oxford University Press, 2009.

[32] Mecklenbr'auker, W. "A tutorial on non-parametric bilinear timefrequency signal representations." Time and frequency representation of signals and systems. Springer Vienna, 1989. 11-68.

[33] J. Mikulka and S. Hanus. "CCK and barker coding implementation in IEEE 802.11 b standard.” In Radioelektronika, 2007. 17th International Conference, pp. 1-4. IEEE, 2007.

[34] S. Narayanan, P.S. Cho and R.J. Marks II, "Three-dimensional seed reconstruction from an incomplete data set for prostate brachytherapy," Phys. Med. Biol., vol.49, pp.3483-3494 (2004).

[35] D.O. North. "An analysis of the factors which determine signal/noise discrimination in pulsed-carrier systems." Proceedings of the IEEE 51.7 (1963): 1016-1027. 
[36] S. Oh and R.J. Marks II, "Some properties of the generalized time frequency representation with cone shaped kernels," IEEE Transactions on Signal Processing, vol.40, No.7, pp.1735-1745, 1992.

[37] S.Oh, R.J. Marks II and L.E. Atlas, "Kernel synthesis for generalized time-frequency distributions using the method of alternating projections onto convex sets," IEEE Transactions on Signal Processing, vol. 42, No.7, July 1994, pp.1653-1661.

[38] Jorge Ojeda- Castañeda, L. R. Berriel-Valdos, and Emma Montes. "Ambiguity function as a design tool for high focal depth." Applied optics 27, no. 4 (1988): 790-795. bibitemPapoulis1974 Athanasios Papoulis. "Ambiguity function in Fourier optics." JOSA 64, no. 6 (1974): 779788.

[39] Mark A. Richards, Fundamentals of Radar Signal Processing, Tata McGraw-Hill Education, 2005.

[40] Mischa Schwartz, William R. Bennett, and Seymour Stein. Communication systems and techniques, John Wiley \& Sons, 1995.

[41] W. W. Siebert, "A Radar Detection Philosophy," Trans. I.R.E., IT-2 204 (1956)

[42] Bernard Sklar, Digital communications, Vol. 2. NJ: Prentice Hall, 2001.

[43] Merrill Ivan Skolnik, Radar handbook (1970).

[44] M. Soumekh, Synthetic aperture radar signal processing (pp. 212-215). New York: Wiley (1999).

[45] Stein, Seymour. "Algorithms for ambiguity function processing." Acoustics, Speech and Signal Processing, IEEE Transactions on 29, no. 3 (1981): 588-599.

[46] George Turin. "An introduction to matched filters." Information Theory, IRE Transactions on 6, no. 3 (1960): 311-329.

[47] R. Turyn and J. Storer. "On binary sequences," Proceedings of the American Mathematical Society 12, no. 3 (1961): 394-399.

[48] Lora G. Weiss, "Wavelets and wideband correlation processing." Signal Processing Magazine, IEEE 11, no. 1 (1994): 13-32.

[49] Philip Mayne Woodward, Probability, Information Theory and Radar, (Pergamon, 1953).

[50] Philip Mayne Woodward, Radar ambiguity analysis. No. RRE-TN-731. ROYAL RADAR ESTABLISHMENT MALVERN (UNITED KINGDOM), 1967. 